\title{
OPEN SETS IN COMPUTABILITY THEORY AND REVERSE MATHEMATICS
}

\author{
DAG NORMANN AND SAM SANDERS
}

\begin{abstract}
To enable the study of open sets in computational approaches to mathematics, lots of extra data and structure on these sets is assumed. For both foundational and mathematical reasons, it is then a natural question, and the subject of this paper, what the influence of this extra data and structure is on the logical and computational properties of basic theorems pertaining to open sets. To answer this question, we study various basic theorems of analysis, like the Baire category, Heine, Heine-Borel, Urysohn, and Tietze theorems, all for open sets given by their (third-order) characteristic functions. Regarding computability theory, the objects claimed to exist by the aforementioned theorems undergo a shift from 'computable' to 'not computable in any type two functional', following Kleene's S1-S9. Regarding Reverse Mathematics, the latter's Main Question, namely which set existence axioms are necessary for proving a given theorem, does not have an unique or unambiguous answer for the aforementioned theorems, working in Kohlenbach's higher-order framework. A finer study of representations of open sets leads to the new ' $\Delta$-functional' which has unique (computational) properties.
\end{abstract}

\section{INTRODUCTION}

Obviousness, much more than beauty, is in the eye of the beholder. For this reason, lest we be misunderstood, we formulate a blanket caveat: all notions (computation, continuity, function, open set, comprehension, et cetera) used in this paper are to be interpreted via their well-known definitions in higher-order arithmetic listed below, unless explicitly stated otherwise.

1.1. Aim and motivation. It is a commonplace that the notion of open set is central to topology and fundamental to large parts of mathematics in ways that few notions can boast. Historical analysis dates back the concept of open set to Baire's 1899 doctoral thesis, while Dedekind already considered this and related concepts twenty years earlier; the associated paper was published much later ([14,31]).

In this paper, we study open sets in computability theory (following Kleene's S1-S9; see Section 2.2) and Reverse Mathematics (RM hereafter; see Section 2.1) in Kohlenbach's higher-order framework. Our motivation -in a nutshell- is that lots of extra data and structure is assumed on open sets in the various 'computational' approaches to mathematics, as detailed in Remark 1.1. For both foundational and mathematical reasons, it is then a natural question, and part of Shore's [51,

Department of Mathematics, The University of Oslo, P.O. Box 1053, Blindern N-0316 OSLO, NORWAY

Department of Mathematics, TU Darmstadt, Germany

E-mail addresses: dnormann@math.uio.no, sasander@me.com. 
Problem 5.1], what the influence of this extra data and structure is on the logical and computational properties of basic theorems pertaining to open sets.

As discussed in detail in Section 1.2, the addition of this extra data and structure has huge consequences for (countable) open-cover compactness, but not for sequential compactness. For instance, the Heine-Borel theorem for countable coverings of closed sets in the unit interval is rather 'mundane' when working with open sets represented via sequences of open balls (and closed sets as complements thereof). Indeed, this second-order theorem is provable from weak König's lemma while the finite sub-covering therein is outright computable (via an unbounded search) in terms of the other data (see [50, IV.1], [29, p. 459], and [8, Lemma 3.13]).

Things are quite different for $\mathrm{HBC}$, the Heine-Borel theorem for countable coverings of closed sets given by third-order (possibly discontinuous) characteristic functions. Indeed, the finite sub-covering from $\mathrm{HBC}$ is no longer computable in any type two functional and the other data, while no system $\Pi_{k}^{1}-C A_{0}^{\omega}$ proves $\mathrm{HBC}$; the former is the higher-order version of $\Pi_{k}^{1}-\mathrm{CA}_{0}$ from Section 2.2. Nonetheless, weak König's lemma plus a small fragmen 1 of countable choice suffices to prove HBC. As discussed in detail in Section 1.2, we may conclude that the Main Question of RM, namely which set existence axioms are necessary for proving the theorem, does not have an unique or unambiguous answer for HBC, but rather depends (heavily) on the presence of countable choice. We obtain similar results for other basic theorems, like the Heine, Urysohn, and Tietze theorems, and the Baire category theorem. We note that the study of the latter theorem requires very different proofs.

We motivate our representation of open sets via third-order characteristic functions by the observation that e.g. $\mathbb{R} \backslash\{0\}$ has an obvious representation via a sequence of open balls, but also a discontinuous characteristic function. In general, open sets are coded in second-order RM by formulas involving an existential numerical quantifier (see [50, II.5.7]), and Kohlenbach has established the intimate connection between these formulas and discontinuous functions in [27, §3]. In this light, the change from RM-codes to characteristic functions only seems like a small step, yet has an immense impact on the Heine-Borel theorem and related theorems.

By the previous, a slightly different representation of open sets can have a huge effect on the associated theorems. It is then a natural question how strong the 'coding principle' is that expresses every characteristic function of an open set can be represented by a sequence of open balls, as well as how hard it is to compute, in the sense of Kleene's S1-S9, this representation. In both cases, the functional $\exists^{3}$ from Section 2.2 suffices, but the latter yields full second-order arithmetic. By contrast, no system $\Pi_{k}^{1}-\mathrm{CA}_{0}^{\omega}$ can prove this coding principle, while no type two functional can compute the countable representation from the other data.

Serendipitously, a finer study of representations of open sets gives rise to the new ' $\Delta$-functional'. In a nutshell, this unqiue functional converts between certain natural representations of open sets; $\Delta$ also has unique computational properties, discussed in detail below, in that it is natural, genuinely type three, but does not add any computational strength to discontinuous functionals like $\exists^{2}$ from Section 2.2 when it comes to computing functions from functions.

\footnotetext{
${ }^{1}$ The small fragment at hand is QF-AC ${ }^{0,1}$ introduced in Section 2.1 which is countable choice restricted to quantifier-free formulas and quantification over Baire space; this fragment is not provable in ZF and is studied in 27.
} 
We discuss the aforementioned results in detail in Section 1.2 We finish this section with a remark on representations of open sets as they are used in various 'computational' approaches to mathematics.

Remark 1.1 (Open sets and representations). A set is open if it contains a neighbourhood around each of its points, and an open set can be written as a countable union of such neighbourhoods in separable spaces. In computational approaches to mathematics, open sets come with various 'constructive' enrichments, as follows.

For instance, the neighbourhood around a point of an open set is often assumed to be given together with this point (see e.g. [5, p. 69]). This is captured by our representation (R.2) in Section 7. Alternatively, open sets are simply represented as countable unions (called 'codes' in [50, II.5.6], 'names' in [61, \$1.3.4], and 'presentations' in [15]) of open neighbourhoods, i.e. a non-deterministic search yields the aforementioned neighbourhood of a point, as in our representation (R.4).

Moreover, there are a number of 'effective' results pertaining to such coded open sets, including the Urysohn lemma and Tietze theorems (see [50, II.7] and [61, §6.2]) in which the object claimed to exist can also be computed (in the sense of Turing) from the inputs. Finally, general results about open sets often require additional computational information, the most common condition being locatedness, which is captured by our representation (R.3) in Section 7 A closed set $A$ is called located if the (continuous) distance function $d(a, A):=\inf _{b \in A} d(a, b)$ exists (see [5, p. 82], [19] or [60, p. 258]). Numerous sufficient conditions are known for the locatedness of (representations of) closed sets ([19]).

1.2. Overview. We discuss the results to be obtained in Sections 3 to 7 in some detail. We assume basic familiarity with RM and computability theory, while we refer to Section 2 for an introduction and the necessary technical details.

First of all, the equivalence between weak König's lemma and the Heine-Borel theorem for countable coverings of the unit interval is one of the early results in second-order RM, announced in 17 and to be found in [50, IV.1.2]. The same equivalence holds if we generalise the latter theorem to closed subsets of the unit interval by [8, Lemma 3.13]. Here, closed sets are the complements of open sets and an open set $U \subset \mathbb{R}$ is represented by some sequence of open balls $\cup_{n \in \mathbb{N}} B\left(a_{n}, r_{n}\right)$ where $a_{n}, r_{n}$ are rationals (see [50, I.4]). We then write the following for any $x \in \mathbb{R}$ :

$$
x \in U \text { if and only if }(\exists n \in \mathbb{N})\left(\left|x-a_{n}\right|<_{\mathbb{R}} r_{n}\right) .
$$

Open and closed sets are well-studied in RM, as is clear from e.g. [8 10].

Secondly, the open $U_{0}=\mathbb{R} \backslash\{0\}$ can (trivially) be represented as in (1.1), but $U_{0}$ has a discontinuous characteristic function. One also readily proves, under the assumption that there exists a discontinuous function on $\mathbb{R}$, that every open set $U$ as in (1.1) has a characteristic function (see [27, §3]). Furthermore, Yu uses sequences of RM-codes to represent discontinuous characteristic functions in 62. In this light, working with third-order (and thus possibly discontinuous) characteristic functions for open sets seems to stay rather close to the representation (1.1) standard in second-order RM, leading to the following definition; see Section 2.1 for $\mathrm{RCA}_{0}^{\omega}$.

Definition 1.2. [Open sets in $\mathrm{RCA}_{0}^{\omega}$ ] We let $Y: \mathbb{R} \rightarrow \mathbb{R}$ represent open subsets of $\mathbb{R}$ as follows: we write ' $x \in Y^{\prime}$ for ' $Y(x)>_{\mathbb{R}} 0$ ' and call a set $Y \subseteq \mathbb{R}$ 'open' if for every $x \in Y$, there is an open ball $B(x, r) \subset Y$ with $r^{0}>0$. A set $Y$ is called 'closed' if the complement, denoted $Y^{c}=\{x \in \mathbb{R}: x \notin Y\}$, is open. 
Note that for open $Y$ as in the previous definition, the formula ' $x \in Y$ ' has the same complexity (modulo higher types) as (1.1), while given $\left(\exists^{2}\right)$ it becomes a 'proper' characteristic function, only taking values ' 0 ' and ' 1 '. Hereafter, an 'open set' refers to Definition 1.2 while 'RM-open set' refers to (1.1). By [50, II.7.1], one can effectively convert between RM-open sets and (RM-codes for) continuous characteristic functions, i.e. (1.1) is included in Definition 1.2

Thirdly, let HBC be the higher-order theorem that for a closed set $C \subseteq[0,1]$ (as in Definition 1.2) a countable covering (consisting of basic open balls) of $C$ has a finite sub-covering. Let $\mathrm{HBC}_{\mathrm{rm}}$ be the second-order theorem based on closed sets as in (1.1). We have the following, where $Z_{2}^{\omega} \equiv \cup_{k} \Pi_{k}^{1}-C A_{0}^{\omega}$ is a higher-order version of $\mathrm{Z}_{2}$ and $\mathrm{HBU}_{\text {closed }}$ is $\mathrm{HBC}$ for uncountable coverings (see Section 2.2 for definitions).

(a) The system $\mathrm{RCA}_{0}$ proves $\mathrm{HBC}_{\mathrm{rm}} \leftrightarrow \mathrm{WKL}$ (see e.g. [8, Lemma 3.13]).

(b) The finite sub-covering in $\mathrm{HBC}_{\mathrm{rm}}$ is computable in terms of the closed set and the countable covering (see [29, §7.3.4]).

(c) The system $Z_{2}^{\omega}$ cannot prove $\mathrm{HBC}$ while $\mathrm{RCA}_{0}^{\omega}+\mathrm{QF}_{-\mathrm{AC}}{ }^{0,1}$ proves $\mathrm{HBC} \leftrightarrow$ WKL and $\mathrm{RCA}_{0}^{\omega}$ proves $\left(\exists^{3}\right) \rightarrow \mathrm{HBU}_{\text {closed }} \rightarrow \mathrm{HBC}$ (Section 3 ).

(d) The finite sub-covering in $\mathrm{HBC}$ is not computable in terms of the closed set, the countable covering, and any type two object (Section 3).

Note that by the final part of item (C), HBC is provable without countable choice, but much stronger comprehension axioms are needed in the absence of the latter. Moreover, since WKL + QF-AC ${ }^{0,1}$ and $\mathrm{HBU}_{\text {closed }}$ are independent but have the same first-order strength, there is no unique set of minimal (comprehension) axioms that proves HBC. Thus, the so-called Main Question of RM from [50, p. 9] does not have an unique or unambiguous answer for HBC. In Sections 3 and 5 we show that the Heine, Urysohn, and Tietze theorems have the same properties. Note that HBC and $Z_{2}^{\omega}$ are third-order in nature, while $Z_{2}^{\Omega}$ is fourth-order in nature.

We stress that the above 'non-standard' behaviour does not apply to sequential compactness: the latter property for closed sets as in Definition[1.2 in the unit interval is equivalent to $\mathrm{ACA}_{0}$ over Kohlenbach's $\mathrm{RCA}_{0}^{\omega}$ by Theorem 3.1 . In other words, changing the coding of open sets does not seem to affect sequential compactness, but does greatly affect countable open-cover compactness.

Fourth, the previous results suggest that Definition [1.2, while quite close to the original (1.1), does provide a stronger notion of open set than (1.1). It is then a natural question how hard it is to prove the 'coding principle' open from Section 4 which expresses that every characteristic function as in Definition 1.2 has a representation in terms of basic open balls as in (1.1). Moreover, one wonders how hard it is to compute (Kleene S1-S9; see Section 2.2) this representation in terms of the other data. We shall provide the following answers.

As shown in Section 4, no type two functional computes such a representation while the functional $\exists^{3}$ suffices; the latter however yields full second-order arithmetic. On the $\mathrm{RM}$ side, no system $\Pi_{k}^{1}-\mathrm{CA}_{0}^{\omega}$ can prove open, while $Z_{2}^{\Omega}$ can. Moreover, $\Pi_{k}^{1}-\mathrm{CA}_{0}^{\omega}+$ open proves (second-order) transfinite recursion for $\Pi_{k}^{1}$-formulas, where the latter is intermediate between $\Pi_{k}^{1}-\mathrm{CA}_{0}$ and $\Pi_{k+1}^{1}-\mathrm{CA}_{0}$. In Section [5, we also show that open is equivalent to the associated Urysohn and Tietze theorems.

Fifth, another prominent theorem about open sets is the Baire category theorem, studied in all the computational approaches to mathematics from Remark 1.1. We therefore study the computational properties of the Baire category theorem in 
Section 6. based on the concept of open set as in Definition 1.2. In this way, a realiser for the Baire category theorem cannot be computed by any type two functional, while the inductive definition operator IND (see Section 6.1) can compute such a realiser, given $\exists^{2}$. We note that the associated proofs in this case are very different from the other proofs in this paper and the known proofs in [35,37.

Sixth, there are of course representations of open sets other than the ones provided by (1.1) and Definition 1.2. We study two such representations, called (R.2) and (R.3) in Section 7 . Intuitively, realisers of the ' $\forall \exists$ '-definition of open sets are the representations as in ( $\underline{R .2}$, while in $(\underline{R .3})$ an open set is given by the function showing that the complement is located. In this context, we study the unique functional $\Delta$ which converts the former to the latter representation. The $\Delta$-functional has surprising computational properties (see Sections 2 and 7 for definitions):

(P1) $\Delta$ is not computable in any type two functional, but computable in any Pincherle realiser, a class weaker than $\Theta$-functionals.

(P2) $\Delta$ is unique, genuinely type three, and adds no computational strength to $\exists^{2}$ in terms of computing functions from functions.

In Section 7.3 we also briefly discuss the computational complexity related to the Baire category theorem and HBC under the representation (R.2) from Section 7.1

We finish this section with a discussion of some of our previous results from [37, which were the starting point of this paper.

Remark 1.3 (The Pincherle phenomenon). Pincherle's theorem is one of the first local-to-global principles, originally proved around 1882 in [40, p. 67], and expresses that a locally bounded function, say on Cantor space, is bounded. Such principles are intimately connected to compactness as claimed by Tao:

Compactness is a powerful property of spaces, and is used in many ways in many different areas of mathematics. One is via appeal to local-to-global principles; one establishes local control on some function or other quantity, and then uses compactness to boost the local control to global control. [54, p. 168]

We have shown in [37] that Pincherle's theorem is closely related to (open-cover) compactness, but has fundamentally different logical and computational properties. For instance, Pincherle's theorem, called PIT ${ }_{o}$ in [37, satisfies the following:

- The system $\mathrm{Z}_{2}^{\omega}$ cannot prove $\mathrm{PIT}_{o}$, while $\mathrm{RCA}_{0}^{\omega}+\mathrm{QF}_{-}-\mathrm{AC}^{0,1}$ proves $\mathrm{WKL} \leftrightarrow$ $\mathrm{PIT}_{o}$ and $\mathrm{RCA}_{0}^{\omega}$ proves $\left(\exists^{3}\right) \rightarrow \mathrm{HBU} \rightarrow \mathrm{PIT}_{o}$.

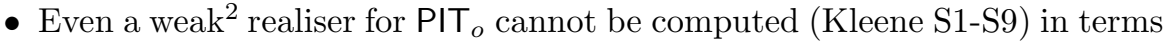
of any type two functional.

Clearly, Pincherle's theorem exhibits the same properties as HBC described in items (IC) and (d) above, and we shall therefore say that HBC exhibits the Pincherle phenomemon, due to Pincherle's theorem PIT $_{o}$ being the first theorem identified as exhibiting the above behaviour, namely in [37. In other words, part of the aim of this paper is to establish the abundance of the Pincherele phenomenon in ordinary mathematics, beyond the few examples from [37.

\footnotetext{
${ }^{2}$ Two kinds of realisers for Pincherle's theorem were introduced in 37]: a weak Pincherle realiser $M_{o}$ takes as input $F^{2}$ that is locally bounded on $2^{\mathbb{N}}$ together with $G^{2}$ such that $G(f)$ is an upper bound for $F$ in $[\bar{f} G(f)]$ for any $f \in 2^{\mathbb{N}}$, and outputs an upper bound $M_{o}(F, G)$ for $F$ on $2^{\mathbb{N}}$. A (normal) Pincherle realiser $M_{u}$ outputs an upper bound $M_{u}(G)$ without access to $F$.
} 
Another way of interpreting the Pincherle phenomenon from Remark 1.3 is as follows: as suggested by its title, it is claimed in [24] that 'disasters' happen in topology in absence of the Axiom of Choice, i.e. working in ZF or weaker fragments of ZFC. We note that [24] mostly deals with countable choice. It is no leap of the imagination to claim that similar disasters already happen for Pincherle's theorem $\mathrm{PIT}_{o}$ and HBC, i.e. in ordinary mathematics. Indeed, Pincherle's theorem and HBC 'should' be equivalent to weak König's lemma, or at least provable from relatively weak axioms, but it seems this can only be guaranteed given countable choice.

\section{Preliminaries}

We introduce Reverse Mathematics in Section 2.1] as well as its generalisation to higher-order arithmetic, and the associated base theory RCA $\mathrm{R}_{0}^{\omega}$. We introduce some essential axioms in Section 2.2

2.1. Reverse Mathematics. Reverse Mathematics is a program in the foundations of mathematics initiated around 1975 by Friedman $([16,17)$ and developed extensively by Simpson ([50]). The aim of RM is to identify the minimal axioms needed to prove theorems of ordinary, i.e. non-set theoretical, mathematics.

We refer to [52] for a basic introduction to RM and to [49,50] for an overview of RM. We expect basic familiarity with RM, but do sketch some aspects of Kohlenbach's higher-order RM ([27]) essential to this paper, including the base theory $\mathrm{RCA}_{0}^{\omega}$ (Definition 2.1). As will become clear, the latter is officially a type theory but can accommodate (enough) set theory via Definition 1.2

First of all, in contrast to 'classical' RM based on second-order arithmetic $\mathrm{Z}_{2}$, higher-order RM uses $\mathrm{L}_{\omega}$, the richer language of higher-order arithmetic. Indeed, while the former is restricted to natural numbers and sets of natural numbers, higher-order arithmetic can accommodate sets of sets of natural numbers, sets of sets of sets of natural numbers, et cetera. To formalise this idea, we introduce the collection of all finite types $\mathbf{T}$, defined by the two clauses:

(i) $0 \in \mathbf{T}$ and (ii) If $\sigma, \tau \in \mathbf{T}$ then $(\sigma \rightarrow \tau) \in \mathbf{T}$,

where 0 is the type of natural numbers, and $\sigma \rightarrow \tau$ is the type of mappings from objects of type $\sigma$ to objects of type $\tau$. In this way, $1 \equiv 0 \rightarrow 0$ is the type of functions from numbers to numbers, and $n+1 \equiv n \rightarrow 0$. Viewing sets as given by characteristic functions, we note that $Z_{2}$ only includes objects of type 0 and 1 .

Secondly, the language $\mathrm{L}_{\omega}$ includes variables $x^{\rho}, y^{\rho}, z^{\rho}, \ldots$ of any finite type $\rho \in \mathbf{T}$. Types may be omitted when they can be inferred from context. The constants of $L_{\omega}$ include the type 0 objects 0,1 and $<_{0},+_{0}, \times_{0},={ }_{0}$ which are intended to have their usual meaning as operations on $\mathbb{N}$. Equality at higher types is defined in terms of ' $={ }_{0}$ ' as follows: for any objects $x^{\tau}, y^{\tau}$, we have

$$
\left[x=_{\tau} y\right] \equiv\left(\forall z_{1}^{\tau_{1}} \ldots z_{k}^{\tau_{k}}\right)\left[x z_{1} \ldots z_{k}={ }_{0} y z_{1} \ldots z_{k}\right]
$$

if the type $\tau$ is composed as $\tau \equiv\left(\tau_{1} \rightarrow \ldots \rightarrow \tau_{k} \rightarrow 0\right)$. Furthermore, $\mathrm{L}_{\omega}$ also includes the recursor constant $\mathbf{R}_{\sigma}$ for any $\sigma \in \mathbf{T}$, which allows for iteration on type $\sigma$-objects as in the special case (2.2). Formulas and terms are defined as usual. One obtains the sub-language $\mathrm{L}_{n+2}$ by restricting the above type formation rule to produce only type $n+1$ objects (and related types of similar complexity).

Definition 2.1. The base theory $\mathrm{RCA}_{0}^{\omega}$ consists of the following axioms. 
(a) Basic axioms expressing that $0,1,<_{0},+_{0}, \times_{0}$ form an ordered semi-ring with equality $=_{0}$.

(b) Basic axioms defining the well-known $\Pi$ and $\Sigma$ combinators (aka $K$ and $S$ in [2]), which allow for the definition of $\lambda$-abstraction.

(c) The defining axiom of the recursor constant $\mathbf{R}_{0}$ : for $m^{0}$ and $f^{1}$ :

$$
\mathbf{R}_{0}(f, m, 0):=m \text { and } \mathbf{R}_{0}(f, m, n+1):=f\left(n, \mathbf{R}_{0}(f, m, n)\right) .
$$

(d) The axiom of extensionality: for all $\rho, \tau \in \mathbf{T}$, we have:

$$
\left(\forall x^{\rho}, y^{\rho}, \varphi^{\rho \rightarrow \tau}\right)\left[x={ }_{\rho} y \rightarrow \varphi(x)={ }_{\tau} \varphi(y)\right] .
$$

(e) The induction axiom for quantifier-free formulas of $\mathrm{L}_{\omega}$.

(f) $\mathrm{QF}-\mathrm{AC}^{1,0}$ : the quantifier-free Axiom of Choice as in Definition 2.2

Note that variables (of any finite type) are allowed in quantifier-free formulas of the language $\mathrm{L}_{\omega}$ : only quantifiers are banned. Moreover, we let $\mathbf{I N D}{ }^{\omega}$ be the induction axiom for all formulas in $\mathrm{L}_{\omega}$.

Definition 2.2. The axiom QF-AC consists of the following for all $\sigma, \tau \in \mathbf{T}$ :

$$
\left(\forall x^{\sigma}\right)\left(\exists y^{\tau}\right) A(x, y) \rightarrow\left(\exists Y^{\sigma \rightarrow \tau}\right)\left(\forall x^{\sigma}\right) A(x, Y(x)),
$$

for any quantifier-free formula $A$ in the language of $\mathrm{L}_{\omega}$.

As discussed in [27, §2], $\mathrm{RCA}_{0}^{\omega}$ and $\mathrm{RCA}_{0}$ prove the same sentences 'up to language' as the latter is set-based and the former function-based. Recursion as in (2.2) is called primitive recursion; the class of functionals obtained from $\mathbf{R}_{\rho}$ for all $\rho \in \mathbf{T}$ is called Gödel's system $T$ of all (higher-order) primitive recursive functionals.

We use the usual notations for natural, rational, and real numbers, and the associated functions, as introduced in [27, p. 288-289].

Definition 2.3 (Real numbers and related notions in $\mathrm{RCA}_{0}^{\omega}$ ).

(a) Natural numbers correspond to type zero objects, and we use ' $n$ ' and ' $n \in \mathbb{N}$ ' interchangeably. Rational numbers are defined as signed quotients of natural numbers, and ' $q \in \mathbb{Q}$ ' and ' $<_{\mathbb{Q}}$ ' have their usual meaning.

(b) Real numbers are coded by fast-converging Cauchy sequences $q_{(\cdot)}: \mathbb{N} \rightarrow$ $\mathbb{Q}$, i.e. such that $\left(\forall n^{0}, i^{0}\right)\left(\left|q_{n}-q_{n+i}\right|<\mathbb{Q} \frac{1}{2^{n}}\right)$. We use Kohlenbach's 'hat function' from [27, p. 289] to guarantee that every $q^{1}$ defines a real number.

(c) We write ' $x \in \mathbb{R}^{\prime}$ to express that $x^{1}:=\left(q_{(\cdot)}^{1}\right)$ represents a real as in the previous item and write $[x](k):=q_{k}$ for the $k$-th approximation of $x$.

(d) Two reals $x, y$ represented by $q_{(\cdot)}$ and $r_{(\cdot)}$ are equal, denoted $x=\mathbb{R} y$, if $\left(\forall n^{0}\right)\left(\left|q_{n}-r_{n}\right| \leq 2^{-n+1}\right)$. Inequality ' $<\mathbb{R}$ ' is defined similarly. We sometimes omit the subscript ' $\mathbb{R}$ ' if it is clear from context.

(e) Functions $F: \mathbb{R} \rightarrow \mathbb{R}$ are represented by $\Phi^{1 \rightarrow 1}$ mapping equal reals to equal reals, i.e. extensionality as in $(\forall x, y \in \mathbb{R})\left(x=\mathbb{R} y \rightarrow \Phi(x)={ }_{\mathbb{R}} \Phi(y)\right)$.

(f) The relation ' $x \leq_{\tau} y$ ' is defined as in (2.1) but with ' $\leq_{0}$ ' instead of ' $=_{0}$ '. Binary sequences are denoted ' $f{ }^{1}, g^{1} \leq_{1} 1$ ', but also ' $f, g \in C$ ' or ' $f, g \in 2^{\mathrm{N}}$ '. Elements of Baire space are given by $f^{1}, g^{1}$, but also denoted ' $f, g \in \mathbb{N}^{\mathbb{N}}$ '.

(g) For a binary sequence $f^{1}$, the associated real in $[0,1]$ is $₫(f):=\sum_{n=0}^{\infty} \frac{f(n)}{2^{n+1}}$.

(h) An object $\mathbf{Y}^{0 \rightarrow \rho}$ is called a sequence of type $\rho$ objects and also denoted $\mathbf{Y}=\left(Y_{n}\right)_{n \in \mathbb{N}}$ or $\mathbf{Y}=\lambda n . Y_{n}$ where $Y_{n}:=\mathbf{Y}(n)$ for all $n^{0}$.

Next, we mention the highly useful ECF-interpretation. 
Remark 2.4 (The ECF-interpretation). The (rather) technical definition of ECF may be found in $[56$, p. $138, \S 2.6]$. Intuitively, the ECF-interpretation $[A]_{\mathrm{ECF}}$ of a formula $A \in \mathrm{L}_{\omega}$ is just $A$ with all variables of type two and higher replaced by type one variables ranging over so-called 'associates' or 'RM-codes' (see [26, §4]); the latter are (countable) representations of continuous functionals. The definition of associate may be found just below Definition 4.4 The ECF-interpretation connects $\mathrm{RCA}_{0}^{\omega}$ and $\mathrm{RCA}_{0}$ (see [27, Prop. 3.1]) in that if $\mathrm{RCA}_{0}^{\omega}$ proves $A$, then $\mathrm{RCA}_{0}$ proves $[A]_{\mathrm{ECF}}$, again 'up to language', as $\mathrm{RCA}_{0}$ is formulated using sets, and $[A]_{\mathrm{ECF}}$ is formulated using types, i.e. using type zero and one objects.

In light of the widespread use of codes in RM and the common practise of identifying codes with the objects being coded, it is no exaggeration to refer to ECF as the canonical embedding of higher-order into second-order arithmetic. For completeness, we list the following notational convention for finite sequences.

Notation 2.5 (Finite sequences). We assume a dedicated type for 'finite sequences of objects of type $\rho^{\prime}$, namely $\rho^{*}$, which we only use for $\rho=0,1$. Since the usual coding of pairs of numbers goes through in $\mathrm{RCA}_{0}^{\omega}$, we shall not always distinguish between 0 and $0^{*}$. Similarly, we do not always distinguish between ' $s^{\rho}$ ' and ' $\left\langle s^{\rho}\right\rangle$ ', where the former is 'the object $s$ of type $\rho$ ', and the latter is 'the sequence of type $\rho^{*}$ with only element $s^{\rho}$ '. The empty sequence for the type $\rho^{*}$ is denoted by ' \langle\rangle$_{\rho}$ ', usually with the typing omitted.

Furthermore, we denote by ' $|s|=n$ ' the length of the finite sequence $s^{\rho^{*}}=$ $\left\langle s_{0}^{\rho}, s_{1}^{\rho}, \ldots, s_{n-1}^{\rho}\right\rangle$, where $|\langle\rangle|=0$, i.e. the empty sequence has length zero. For sequences $s^{\rho^{*}}, t^{\rho^{*}}$, we denote by ' $s * t$ ' the concatenation of $s$ and $t$, i.e. $(s * t)(i)=s(i)$ for $i<|s|$ and $(s * t)(j)=t(|s|-j)$ for $|s| \leq j<|s|+|t|$. For a sequence $s^{\rho^{*}}$, we define $\bar{s} N:=\langle s(0), s(1), \ldots, s(N-1)\rangle$ for $N^{0}<|s|$. For a sequence $\alpha^{0 \rightarrow \rho}$, we also write $\bar{\alpha} N=\langle\alpha(0), \alpha(1), \ldots, \alpha(N-1)\rangle$ for any $N^{0}$. By way of shorthand, $\left(\forall q^{\rho} \in Q^{\rho^{*}}\right) A(q)$ abbreviates $\left(\forall i^{0}<|Q|\right) A(Q(i))$, which is (equivalent to) quantifier-free if $A$ is.

2.2. Higher-order computability theory. As noted above, some of our main results will be proved using techniques from computability theory. Thus, we first make our notion of 'computability' precise as follows.

(I) We adopt ZFC, i.e. Zermelo-Fraenkel set theory with the Axiom of Choice, as the official metatheory for all results, unless explicitly stated otherwise.

(II) We adopt Kleene's notion of higher-order computation as given by his nine clauses S1-S9 (see [29, Ch. 5] or [25]) as our official notion of 'computable'.

Similar to 33 37, one main aim of this paper is the study of functionals of type 3 that are natural from the perspective of mathematical practise. Our functionals are genuinely of type 3 in the sense that they are not computable from any functional of type 2 . The following definition ${ }^{3}$ is then standard in this context.

Definition 2.6. A functional $\Phi^{3}$ is countably based if for every $F^{2}$ there is countable $X \subset \mathbb{N}^{\mathbb{N}}$ such that $\Phi(F)=\Phi(G)$ for every $G$ that agrees with $F$ on $X$.

Now, if $\Phi^{3}$ is computable in a functional of type 2 , then it is countably based, but the converse does not hold. However, Hartley proves in 20] that, assuming

\footnotetext{
${ }^{3}$ Stanley Wainer (unpublished) has defined the countably based functionals of finite type as an analogue of the continuous functionals, while John Hartley has investigated the computability theory of this type structure (see [20]). We will only need the simple cases of countably based functionals of type level at most 3 in this paper.
} 
the Axiom of Choice and the Continuum Hypothesis, if $\Phi^{3}$ is not countably based, then there is some $F^{2}$ such that $\exists^{3}$ (see below) is computable in $\Phi$ and $F$. In other words, stating the existence of such $\Phi$ brings us 'close to' $Z_{2}^{\Omega}$ (see below). In the sequel, we shall explicitly point out where we use countably based functionals.

For the rest of this section, we introduce some existing functionals which will be used below. In particular, we introduce some functionals which constitute the counterparts of second-order arithmetic $\mathbf{Z}_{2}$, and some of the Big Five systems, in higher-order RM. We use the formulation from [27, 35].

First of all, $\mathrm{ACA}_{0}$ is readily derived from:

$$
\begin{aligned}
\left(\exists \mu^{2}\right)\left(\forall f^{1}\right)[(\exists n)(f(n)=0) \rightarrow[(f(\mu(f))=0) & \wedge(\forall i<\mu(f)) f(i) \neq 0] \quad\left(\mu^{2}\right) \\
& \wedge[(\forall n)(f(n) \neq 0) \rightarrow \mu(f)=0]],
\end{aligned}
$$

and $\mathrm{ACA}_{0}^{\omega} \equiv \mathrm{RCA}_{0}^{\omega}+\left(\mu^{2}\right)$ proves the same sentences as $\mathrm{ACA}_{0}$ by [23, Theorem 2.5]. The (unique) functional $\mu^{2}$ in $\left(\mu^{2}\right)$ is also called Feferman's $\mu$ ([2]), and is clearly discontinuous at $f={ }_{1} 11 \ldots$; in fact, $\left(\mu^{2}\right)$ is equivalent to the existence of $F: \mathbb{R} \rightarrow \mathbb{R}$ such that $F(x)=1$ if $x>_{\mathbb{R}} 0$, and 0 otherwise $([27, \S 3])$, and to

$$
\left(\exists \varphi^{2} \leq_{2} 1\right)\left(\forall f^{1}\right)[(\exists n)(f(n)=0) \leftrightarrow \varphi(f)=0] .
$$

Secondly, $\Pi_{1}^{1}-\mathrm{CA}_{0}$ is readily derived from the following sentence:

$$
\left(\exists \mathrm{S}^{2} \leq_{2} 1\right)\left(\forall f^{1}\right)\left[\left(\exists g^{1}\right)\left(\forall n^{0}\right)(f(\bar{g} n)=0) \leftrightarrow \mathrm{S}(f)=0\right],
$$

and $\Pi_{1}^{1}-\mathrm{CA}_{0}^{\omega} \equiv \mathrm{RCA} A_{0}^{\omega}+\left(\mathrm{S}^{2}\right)$ proves the same $\Pi_{3}^{1}$-sentences as $\Pi_{1}^{1}-\mathrm{CA}_{0}$ by 41 , Theorem 2.2]. The (unique) functional $\mathrm{S}^{2}$ in $\left(\mathrm{S}^{2}\right)$ is also called the Suslin functional ([27). By definition, the Suslin functional $S^{2}$ can decide whether a $\Sigma_{1}^{1}$-formula as in the left-hand side of $\left(\mathrm{S}^{2}\right)$ is true or false. We similarly define the functional $S_{k}^{2}$ which decides the truth or falsity of $\Sigma_{k}^{1}$-formulas; we also define the system $\Pi_{k}^{1}$ - $C A_{0}^{\omega}$ as $\mathrm{RCA}_{0}^{\omega}+\left(\mathrm{S}_{k}^{2}\right)$, where $\left(\mathrm{S}_{k}^{2}\right)$ expresses that $\mathrm{S}_{k}^{2}$ exists. Note that we allow formulas with function parameters, but not functionals here. In fact, Gandy's Superjump (18]) constitutes a way of extending $\Pi_{1}^{1}-\mathrm{CA}_{0}^{\omega}$ to parameters of type two. We identify the functionals $\exists^{2}$ and $\mathrm{S}_{0}^{2}$ and the systems $\mathrm{ACA}_{0}^{\omega}$ and $\Pi_{k}^{1}-\mathrm{CA} \mathrm{A}_{0}^{\omega}$ for $k=0$. We note that the operators $\nu_{n}$ from [11, p. 129] are essentially $S_{n}^{2}$ strengthened to return a witness (if existant) to the $\Sigma_{k}^{1}$-formula at hand.

Thirdly, full second-order arithmetic $Z_{2}$ is readily derived from $\cup_{k} \Pi_{k}^{1}-\mathrm{CA}_{0}^{\omega}$, or from:

$$
\left(\exists E^{3} \leq_{3} 1\right)\left(\forall Y^{2}\right)\left[\left(\exists f^{1}\right)(Y(f)=0) \leftrightarrow E(Y)=0\right],
$$

and we therefore define $Z_{2}^{\Omega} \equiv \mathrm{RCA}_{0}^{\omega}+\left(\exists^{3}\right)$ and $Z_{2}^{\omega} \equiv \cup_{k} \Pi_{k}^{1}-\mathrm{CA}_{0}^{\omega}$, which are conservative over $Z_{2}$ by [23, Cor. 2.6]. Despite this close connection, $Z_{2}^{\omega}$ and $Z_{2}^{\Omega}$ can behave quite differently, as discussed in e.g. $35, \S 2.2]$. The functional from $\left(\exists^{3}\right)$ is also called ' $\exists^{3}$ ', and we use the same convention for other functionals.

Fourth, the Heine-Borel theorem states the existence of a finite sub-covering for an open covering of certain spaces. Now, a functional $\Psi: \mathbb{R} \rightarrow \mathbb{R}^{+}$gives rise to the canonical covering $\cup_{x \in I} I_{x}^{\Psi}$ for $I \equiv[0,1]$, where $I_{x}^{\Psi}$ is the open interval $(x-\Psi(x), x+\Psi(x))$. Hence, the uncountable covering $\cup_{x \in I} I_{x}^{\Psi}$ has a finite subcovering by the Heine-Borel theorem; in symbols:

$$
\left(\forall \Psi: \mathbb{R} \rightarrow \mathbb{R}^{+}\right)\left(\exists y_{1}, \ldots, y_{k} \in I\right)(\forall x \in I)\left(x \in \cup_{i \leq k} I_{y_{i}}^{\Psi}\right) .
$$

Note that HBU is almost verbatim Cousin's lemma ([13, p. 22]), i.e. the HeineBorel theorem restricted to canonical coverings. This restriction does not make a 
big difference, as shown in [44]. By [35, 37, $Z_{2}^{\Omega}$ proves HBU but $Z_{2}^{\omega}+$ QF-AC ${ }^{0,1}$ cannot, and basic properties of the gauge integral ([32,53]) are equivalent to HBU.

Fifth, since Cantor space (denoted $C$ or $2^{\mathbb{N}}$ ) is homeomorphic to a closed subset of $[0,1]$, the former inherits the same property. In particular, for any $G^{2}$, the corresponding 'canonical covering' of $2^{\mathbb{N}}$ is $\cup_{f \in 2^{\mathbb{N}}}[\bar{f} G(f)]$ where $\left[\sigma^{0^{*}}\right]$ is the set of all binary extensions of $\sigma$. By compactness, there are $f_{0}, \ldots, f_{n} \in 2^{\mathbb{N}}$ such that the set of $\cup_{i \leq n}\left[\bar{f}_{i} G\left(f_{i}\right)\right]$ still covers $2^{\mathbb{N}}$. By [35, Theorem 3.3], HBU is equivalent to the same compactness property for $C$, as follows:

$$
\left(\forall G^{2}\right)\left(\exists f_{1}, \ldots, f_{k} \in C\right)(\forall f \in C)\left(f \in \cup_{i \leq k}\left[\overline{f_{i}} G\left(f_{i}\right)\right]\right) .
$$

We now introduce the specification $\operatorname{SFF}(\Theta)$ for a (non-unique) functional $\Theta$ which computes a finite sequence as in $\mathrm{HBU}_{\mathrm{c}}$. We refer to such a functional $\Theta$ as a realiser for the compactness of Cantor space, and simplify its type to ' 3 '.

$$
\left(\forall G^{2}\right)\left(\forall f^{1} \leq_{1} 1\right)(\exists g \in \Theta(G))(f \in[\bar{g} G(g)]) .
$$

Clearly, there is no unique such $\Theta$ (just add more binary sequences to $\Theta(G)$ ) and any functional satisfying the previous specification is referred to as a ' $\Theta$-functional' or a 'special fan functional'. As to their provenance, $\Theta$-functionals were introduced as part of the study of the Gandy-Hyland functional in [43, §2] via a slightly different definition. These definitions are identical up to a term of Gödel's $T$ of low complexity by [34, Theorem 2.6].

Sixth, a number of higher-order axioms are studied in [46] including the following comprehension axiom (see also Remark 2.7):

$$
\left(\forall Y^{2}\right)(\exists X \subset \mathbb{N})(\forall n \in \mathbb{N})\left(n \in X \leftrightarrow\left(\exists f^{1}\right)(Y(f, n)=0)\right) .
$$

We only mention that this axiom is equivalent to e.g. the monotone convergence theorem for nets indexed by Baire space (see [46, §3]). As it turns out, the coding principle open (see Section 4) is closely related to BOOT and fragments, as shown in Section 4.1. Finally, we mention some historical remarks related to BOOT.

Remark 2.7 (Historical notes). First of all, BOOT is called the 'bootstrap' principle as it is weak in isolation (equivalent to $A C A_{0}$ under ECF, in fact), but becomes much stronger when combined with comprehension axioms: $\Pi_{k}^{1}-\mathrm{CA}_{0}^{\omega}+\mathrm{BOOT}$ readily proves $\Pi_{k+1}^{1}-\mathrm{CA}_{0}$.

Secondly, BOOT is definable in Hilbert-Bernays' system $H$ from the Grundlagen der Mathematik (see [22, Supplement IV]). In particular, one uses the functional $\nu$ from [22, p. 479] to define the set $X$ from BOOT. In this way, BOOT and subsystems of second-order arithmetic can be said to 'go back' to the Grundlagen in equal measure, although such claims may be controversial.

Thirdly, after the completion of [4], it was observed by the second author that Feferman's 'projection' axiom (Proj1) from [15] is similar to BOOT. The former is however formulated using sets (and set parameters), which makes it more 'explosive' than BOOT in that full $Z_{2}$ follows when combined with $\left(\mu^{2}\right)$, as noted in [15, I-12].

\section{The Heine-Borel theorem}

3.1. Introduction and preliminaries. In this section, we establish the results sketched in Section 1.2 for the (countable) Heine-Borel theorem and related (countable) Vitali covering theorem. Hereafter, we use the notion of open (and closed) set as outlined in Definition 1.2, unless explicitly stated otherwise. 
In particular, we show that sequential compactness behaves 'as normal' in this section, but that Heine-Borel compactness for countable coverings by intervals now behaves quite out of the ordinary, namely that it suffers from the Pincherle phenomenon (see Section 3.2). In Section 3.3 we establish the computability-theoretic results in item (ㄷ) from Section 1.2 and some related results. All the aforementioned deals with countable coverings consisting of open intervals, and we study coverings consisting of arbitrary open sets in Section 3.4. The latter results are complimentary to the rest and endow our equivalences with a certain robustness.

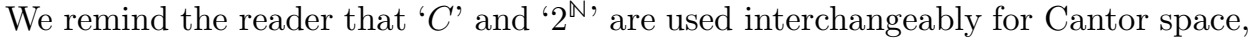
but this use is always carefully separated from the generic term 'closed set $C \subseteq \mathbb{R}$ '.

First of all, Theorem 3.1] is a sanity check for Definition 1.2. our closed sets have the same properties as RM-codes for closed sets, as follows.

(a) RM-closed sets are sequentially closed, i.e. if a sequence in an RM-closed set converges to some limit, the latter is also in the set (trivial in $\mathrm{RCA}_{0}$ ).

(b) RM-closed sets in $[0,1]$ are sequentially compact in $\mathrm{ACA}_{0}$ ([8, Lemma 3.14]).

(c) Given a sequence in an RM-closed set in $[0,1], \exists^{2}$ computes the limit (41]). The following theorem shows that our closed sets mirror these three items perfectly.

Theorem 3.1. The system $\mathrm{RCA}_{0}^{\omega}$ proves that a closed set is sequentially closed. The system $\mathrm{RCA}_{0}^{\omega}$ proves the equivalence between $\mathrm{ACA}_{0}$ and the statement a closed set in $[0,1]$ is sequentially compact. The functional $\exists^{2}$ computes an accumulation point of a sequence in a closed set in $[0,1]$.

Proof. For the first part, if a sequence $\lambda n . x_{n}$ in a closed set $C \subset \mathbb{R}$ converges to $y \in \mathbb{R}$, but $y \notin C$, then there is $N^{0}$ such that $B\left(y, \frac{1}{2^{N}}\right) \subset C^{c}$, as the complement of $C$ is open. However, $x_{n}$ is eventually in $B\left(y, \frac{1}{2^{N}}\right)$ by definition, a contradiction.

For the second part, the reversal follows from considering the unit interval and [50, III.2.2]. For the forward direction, the usual proof of the Bolzano-Weierstrass theorem as in [50, III.2.1] goes through, modulo using $\left(\exists^{2}\right)$ to decide elementhood of closed sets. In case $\neg\left(\exists^{2}\right)$, all $\mathbb{R} \rightarrow \mathbb{R}$-functions are continuous by [27, §3] and closed sets in $[0,1]$ reduce to the usual RM-definition by [50, II.5.7] and [26, Prop. 4.10]. The second-order proof from [8, Lemma 3.14] now finishes this case. The law of excluded middle $\left(\exists^{2}\right) \vee \neg\left(\exists^{2}\right)$ finishes the proof.

The previous theorem can be generalised to other theorems pertaining to sequential compactness (see e.g. [50, III]), like the monotone convergence theorem for sequences in closed subsets of the unit interval.

3.2. Heine-Borel theorem for basic coverings. Theorem 3.1 shows that our notion of closed sets has the usual properties when it comes to sequential compactness. In this section, we show that the situation is markedly different for Heine-Borel compactness: Theorems 3.3 and 3.5 namely show that HBC, defined as follows, suffers from the Pincherle phenomenon.

Definition 3.2. [HBC] Let $C \subseteq[0,1]$ be closed and let $\left(a_{n}\right)_{n \in \mathbb{N}},\left(b_{n}\right)_{n \in \mathbb{N}}$ be sequences of reals with $C \subseteq \cup_{n \in \mathbb{N}}\left(a_{n}, b_{n}\right)$. Then $C \subseteq \cup_{n \leq n_{0}}\left(a_{n}, b_{n}\right)$ for some $n_{0} \in \mathbb{N}$.

We let $\mathrm{HBC}_{\mathrm{rm}}$ be $\mathrm{HBC}$ with $C$ represented by RM-codes. By Theorem 3.3, HBC is provable without countable choice and has weak first-order strength. Indeed, 
$\mathrm{HBU}_{\text {closed }}$ is $\mathrm{HBU}$ generalised to closed sets $C \subseteq[0,1]$, and both have the firstorder strength of WKL; this follows from applying ECF and noting [8, Lemma 3.13]. Furthermore, $\mathrm{Z}_{2}^{\Omega}$ proves $\mathrm{HBU}_{\text {closed }}$ in the same way as in [37, Theorem 4.2].

Theorem 3.3. Both $\mathrm{RCA}_{0}^{\omega}+\mathrm{WKL}+\mathrm{QF}-\mathrm{AC}^{0,1}$ and $\mathrm{RCA}_{0}^{\omega}+\mathrm{HBU}_{\text {closed }}$ prove $\mathrm{HBC}$.

Proof. For the first part, in case $\neg\left(\exists^{2}\right)$, all functions on $\mathbb{R}$ are continuous by [27, §3]. Following the results in [26, §4], continuous functions on $[0,1]$ have an RM-code, i.e. our definition of open set reduces to an $\mathrm{L}_{2}$-formula in $\Sigma_{1}^{0}$, which (equivalently) defines a code for an open set by [50, II.5.7]. In this way, $\mathrm{HBC}$ is merely $\mathrm{HBC}_{\mathrm{rm}}$, which follows from WKL by [8, Lemma 3.13]. In case $\left(\exists^{2}\right)$, let $C \subseteq[0,1]$ be a closed set and let $\left(a_{n}\right)_{n \in \mathbb{N}},\left(b_{n}\right)_{n \in \mathbb{N}}$ be as in HBC. If there is no finite sub-covering, then $\left(\forall m^{0}\right)(\exists x \in C)\left[x \notin \cup_{n \leq m}\left(a_{n}, b_{n}\right)\right]$. Apply QF-AC ${ }^{0,1}$ and $\left(\exists^{2}\right)$ to obtain a sequence $\lambda n . x_{n}$ of reals in $C$ with this property. Since $\left(\exists^{2}\right) \rightarrow \mathrm{ACA}_{0}$, any sequence in $[0,1]$ has a convergent sub-sequence $\lambda n . y_{n}$ by [50, III.2]. If $y_{n}$ converges to $y \notin C$, then there is $N^{0}$ such that $B\left(y, \frac{1}{2^{N}}\right) \subset C^{c}$, as the complement of $C$ is open by definition. However, $y_{n}$ is eventually in $B\left(y, \frac{1}{2^{N}}\right)$ by definition, a contradiction. Hence, $\lim _{n \rightarrow \infty} y_{n}=y \in C$ but if $y \in\left(a_{k}, b_{k}\right)$, then $y_{n}$ is eventually in this interval, which contradicts the definition of $x_{n}$ (and $y_{n}$ ). The law of excluded middle now finishes the proof.

For the second part, let $C \subseteq[0,1]$ be a closed set and let $a_{n}, b_{n}$ as in HBC. Similar to the first case, we may assume $\left(\exists^{2}\right)$. Apply QF-AC ${ }^{1,0}$ and $\left(\exists^{2}\right)$ to $(\forall x \in$ $C)\left(\exists n^{0}\right)\left(x \in\left(a_{n}, b_{n}\right)\right)$ to obtain $\Psi^{2}$ yielding $n^{0}$ from $x \in C$. Then $\cup_{x \in C}\left(a_{\Psi(x)}, b_{\Psi(x)}\right)$ is a covering of $C$ that is readily converted to a canonical covering. We now obtain $\mathrm{HBC}$ from applying $\mathrm{HBU}_{\text {closed }}$.

Corollary 3.4. The system $\mathrm{RCA}_{0}^{\omega}+\mathrm{QF}-\mathrm{AC}^{0,1}$ proves $\mathrm{WKL} \leftrightarrow \mathrm{HBC}$.

Proof. The reverse direction follows from [50, II.7.1] as the latter provides (RMcodes for) continuous characteristic functions for RM-open sets (and vice versa). These codes become type two functionals by QF-AC ${ }^{1,0}$, included in $\mathrm{RCA}_{0}^{\omega}$. Alternatively, use $C=[0,1]$ and the results in [50, IV.1].

In contrast to sequential compactness and Theorem 3.1, a proof of countable Heine-Borel compactness as in HBC seems to either require countable choice or lots of comprehension, i.e. we observe the Pincherle phenomenon.

Theorem 3.5. The system $Z_{2}^{\omega}$ cannot prove $\mathrm{HBC}$.

Proof. We will modify the method used to prove [35, Theorem 3.4] and [37, Theorem 4.8]. As in those proofs, let $A=\cup_{k \in \mathbb{N}} A_{k}$ be a countable subset of $\mathbb{N}^{\mathbb{N}}$ defined by the following three conditions.

(i) We have that $\Pi_{n}^{1}$-formulas are absolute for $\left(A, \mathbb{N}^{\mathbb{N}}\right)$ for all $n$.

(ii) For all $k$, we have $A_{k} \subseteq A_{k+1}$ and there is $f_{k} \in A_{k+1}$ enumerating $A_{k}$.

(iii) There is a sequence $g_{1}, g_{2}, \ldots$ in $A$ such that for all $k$ there is a number $n_{k}$ such that $A_{k}$ is the computational closure of $g_{0}, \ldots, g_{n_{k}}$ relative to $S_{k}^{2}$.

In a nutshell, we shall define a model $\mathcal{M}$ and a set $X$ therein, such that the former thinks the latter is closed. We also identify a countable covering $\cup_{n \in \mathbb{N}}\left(a_{n}, b_{n}\right)$ of $X$ that has no finite sub-covering inside $\mathcal{M}$, i.e. $\mathrm{HBC}$ fails in $\mathcal{M}$.

Let $\mathbf{C} \subset[0,1]$ be the classical Cantor set (available in $\mathrm{RCA}_{0}$ by [50, I.8.6]) and let $\left\{\left(a_{i}, b_{i}\right)\right\}_{i \in \mathbb{N}}$ be a computable enumeration of the set of open intervals forming 
the complement of $\mathbf{C}$. Fix an element $x \in \mathbf{C}$ that has no code in $A$. For each $k$, define $x_{k}$ as an element with a code in $A_{k+1} \backslash A_{k}$ such that $x_{k} \notin \mathbf{C}$ and such that the distance from $x$ to $x_{k}$ is bounded by $2^{-k}$. This construction is possible as the set of elements in $[0,1]$ with codes in $A_{k+1} \backslash A_{k}$ is dense.

Define $X=\left\{x_{k}: k \in \mathbb{N}\right\}$ and consider the type-structure $\mathcal{M}$ obtained by closing $A$ and $X$ under computability relative to all $\mathrm{S}_{k}^{2}$. In computations relative to $\mathrm{S}_{k}^{2}$ and $X$, the two sets $X$ and $\left\{x_{l}: l<k\right\}$ will give the same oracle answers, so $A_{k}$ will be closed under computability relative to $S_{k}^{2}$ and $X$.

We now show that $X$ is a closed set from the point of view of $\mathcal{M}$. To this end, consider $y \in([0,1] \backslash X)$ with a code in $A$. Fix $\epsilon:=\mid x-y]>0$ and let $k$ be so large that $2^{k-1}<\epsilon$. Then at most $x_{i}$ for $i<k$ will be in $\epsilon$-distance from $y$ and thus $y$ has a positive distance to $X$. In this way, $\mathcal{M}$ satisfies that $X$ is closed.

Finally, the computable family $\left\{\left(a_{i}, b_{i}\right)\right\}_{i \in \mathbb{N}}$ is in $\mathcal{M}$ and covers $X$. Now, in the real world, $X$ has a cluster point in $\mathbf{C}$ which is different from all $a_{i}$ and $b_{i}$. Hence, there cannot be a finite sub-covering of $X$, and there is also no finite sub-covering of $X$ in $\mathcal{M}$. As a result, $\mathrm{HBC}$ fails in $\mathcal{M}$ and we are done.

Corollary 3.6. A proof of $\mathrm{HBC}$ goes through in $\mathrm{WKL}+\mathrm{QF}-\mathrm{AC}^{0,1}$ or $\mathrm{Z}_{2}^{\Omega}$, while $\mathrm{Z}_{2}^{\omega}$ does not suffice.

The negative results from the theorem also yield the following nice disjunction.

Corollary 3.7. The system $\mathrm{RCA}_{0}^{\omega}$ proves $\mathrm{WKL}_{0} \leftrightarrow\left[\mathrm{ACA}_{0} \vee \mathrm{HBC}\right]$.

Proof. The reverse implications are immediate by the previous. The forward implication follows from $\left(\exists^{2}\right) \vee \neg\left(\exists^{2}\right)$ and the proof of Theorem 3.3 ,

The previous results are not an isolated incident, as witnessed by the following. Note that items (b) to $(\mathbb{E})$ are studied in [50, VI.2] for RM-codes of closed sets,

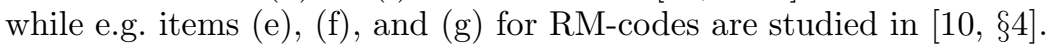

Theorem 3.8. The following theorems imply $\mathrm{HBC}$ over $\mathrm{RCA}_{0}^{\omega}$ :

(a) Pincherle's theorem for [0,1]: a locally bounded function on $[0,1]$ is bounded.

(b) If $F^{2}$ is continuous on a closed set $D \subset 2^{\mathbb{N}}$, it is bounded on $D$.

(c) If $F^{2}$ is continuous on a closed set $D \subset 2^{\mathbb{N}}$, it is uniformly cont. on $D$.

(d) If $F$ is continuous on a closed set $D \subset[0,1]$, it is bounded on $D$.

(e) If $F$ is continuous on a closed set $D \subset[0,1]$, it is uniformly cont. on $D$.

(f) If $F$ is continuous on a closed set $D \subset[0,1]$, it attains a maximum on $D$.

(g) If $F$ is continuous on a closed set $D \subset[0,1]$, then for every $\varepsilon>0$ there is a polynomial $p(x)$ such that $|p(x)-F(x)|<\varepsilon$ for all $x \in D$.

Proof. By the results in [37, §4] and [50, IV], all items from the theorem imply WKL. Moreover, in case $\neg\left(\exists^{2}\right)$, closed sets reduce to RM-codes for closed sets, i.e. $\mathrm{HBC}$ is just $\mathrm{HBC}_{\mathrm{rm}}$, which follows from $\mathrm{WKL}$ by [8, Lemma 3.13]. Similarly, items (b) and (C) reduce to their second-order counterparts, equivalent to $\mathrm{WKL}_{0}$ by [50, IV.2]. Hence, we may assume $\left(\exists^{2}\right)$ in the following. For item (国), let $a_{n}, b_{n}$ be as in the antecedent of HBC, i.e. $(\forall x \in C)\left(\exists n^{0}\right)\left(x \in\left(a_{n}, b_{n}\right)\right)$. Applying QF-AC ${ }^{1,0}$ and $\left(\exists^{2}\right)$ (to decide whether $x \in C$ or not), one obtains $\Phi^{2}$ such that $\Phi(x)$ is the least such $n$ if $x \in C$. By definition, $\Phi$ satisfies $\Phi(y) \leq \Phi(x)$ for any $x \in C$, $y \in C \cap B(x, r)$, and small enough $r>_{\mathbb{R}} 0$. Hence, the function $f: \mathbb{R} \rightarrow \mathbb{R}$ defined as $\Phi(x)$ if $x \in C$ and 1 otherwise, is locally bounded on [0,1]. By Pincherle's theorem, 
$f$ is bounded on $[0,1]$, implying that $\Phi$ is bounded on $C$ and immediately yielding a finite sub-covering for $\cup_{n \in \mathbb{N}}\left(a_{n}, b_{n}\right)$, as required for $\mathrm{HBC}$.

For item (b), since Cantor space is homeomorphic to a closed subset of $[0,1]$, $\mathrm{HBC}$ is equivalent to $\mathrm{HBC}$ for Cantor space. Let $D \subseteq 2^{\mathbb{N}}$ be closed and let $\lambda n . \sigma_{n}^{0 \rightarrow 0^{*}}$ be a sequence of finite binary sequences covering $D$, i.e. $(\forall f \in D)\left(\exists n^{0}\right)\left(f \in\left[\sigma_{n}\right]\right)$. Applying QF-AC ${ }^{1,0}$ and $\left(\exists^{2}\right)$ (to decide whether $x \in D$ or not), one obtains $\Phi^{2}$ such that $\Phi(f)$ is the least such $n$ if $x \in D$. Define $G(f):=\left|\sigma_{\Phi(f)}\right|$ and note:

$$
\left(\forall f, g \in 2^{\mathbb{N}}\right)(\bar{f} G(f)=\bar{g} G(f) \rightarrow \Phi(f)=\Phi(g)),
$$

i.e. $\Phi$ is continuous with modulus of continuity $G$. Item (b) proves that $\Phi$ is bounded on $D$, yielding a finite sub-covering of $\cup_{n \in \mathbb{N}}\left[\sigma_{n}\right]$. Item (IC) now also readily implies HBC. For items (d) and (还), since closed sets in Cantor space are also closed sets in $[0,1]$, these items follows from the items (B) and (ㄷ). Finally, note that either of items (마) or (g) imply item (d).

Note that by the theorem, the Tietze (extension) theorem for closed sets as in Definition [1.2 is not provable in $Z_{2}^{\omega}$. We establish sharper results in Section 5 . Moreover, $\mathrm{ACA}_{0} \leftrightarrow\left[\left(\exists^{2}\right) \vee(\right.$ item $\left.(\mathbb{f}))\right]$ follows in the same way as for Corollary 3.7 .

Finally, regarding (3.1), Kohlenbach shows in [26, §4] that over $\mathrm{RCA}_{0}^{\omega}$ the existence of a modulus of continuity is equivalent to the existence of an RM-code, i.e. the exact formulation of continuity does not matter in the previous theorem.

3.3. Computability theory and related results. We establish the results in item (d) from Section 1.2 pertaining to HBC, and discuss related results, including the weaker Vitali covering lemma. We define a realiser for $\mathrm{HBC}$ as follows.

Definition 3.9. A functional $\beta^{3}$ is called a realiser for $\mathrm{HBC}$ if for closed $C \subset[0,1]$ and sequences of rationals $\left(a_{n}\right)_{n \in \mathbb{N}},\left(b_{n}\right)_{n \in \mathbb{N}}$ such that $C \subseteq \cup_{n \in \mathbb{N}}\left(a_{n}, b_{n}\right)$, we also have $C \subseteq \cup_{n \leq \beta\left(C, a_{n}, b_{n}\right)}\left(a_{n}, b_{n}\right)$.

The following theorem is not that surprising in light of some of our previous results. We shall establish a more impressive result in Theorem 7.9 .

Theorem 3.10. No type two functional can compute a realiser $\beta^{3}$ for HBC.

Proof. The functional $\beta$ is easily seen to be not countably based, namely as follows. Now put $D=\left[\frac{1}{3}, \frac{2}{3}\right], a_{n}=\frac{1}{n}$ and $b_{n}=1$. If $X$ is a countable base for the value $\beta\left(D, a_{n}, b_{n}\right)=k$, we may choose $x \notin X$ in the interval $\left(0, \frac{1}{k}\right)$ and obtain a contradiction by considering the set $D^{\prime}=D \cup\{x\}$.

Finally, it is a natural question whether Theorem 3.5 holds for weaker principles, e.g. from the RM of WWKL; the latter is the restriction of WKL to trees of positive measure and equivalent to Vitali's covering theorem for countable coverings (see [50, X.1]). Towards a positive answer, consider the following covering theorem WHBC. Note that the latter is just [42, Lemma 8, item 2] formulated using Definition 1.2

Definition 3.11. [WHBC] Let $\varepsilon>0$, let $C \subseteq[0,1]$ be a closed set, and let $\left(a_{n}\right)_{n \in \mathbb{N}}$, $\left(b_{n}\right)_{n \in \mathbb{N}}$ be sequences of reals such that $C \subseteq \cup_{n \in \mathbb{N}}\left(a_{n}, b_{n}\right)$. Then there are $n_{0} \in \mathbb{N}$ and reals $c_{0}, d_{0}, \ldots, c_{m_{0}}, d_{m_{0}}$ such that $C \subseteq \bigcup_{n \leq n_{0}}\left(a_{n}, b_{n}\right) \cup \bigcup_{m \leq m_{0}}\left(c_{m}, d_{m}\right)$ and $\sum_{m \leq m_{0}}\left|c_{m}-d_{m}\right|<\varepsilon$. 
On one hand, ECF clearly converts WHBC into a theorem at the level of WWKL. On the other hand, the equivalence WWKL $\leftrightarrow$ WHBC can be proved as in Corollary 3.4 additionally assuming the coding principle that every continuous functional on Baire space has a code (see [26, §4] for this coding principle). We have the following improvement of Theorem 3.5 .

Theorem 3.12. The system $Z_{2}^{\omega}$ cannot prove $\mathrm{WHBC}$

Proof. The following proof is a (considerable) variation on the proof of Theorem 3.5 ,

First of all, we provide a sketch, as follows: let $A, A_{k}$ be as in the proof of Theorem 3.5. Analogously, we shall define a certain sequence $\left\{x_{k}\right\}_{k \in \mathbb{N}}$ of codes in $A$ such that $x_{k}$ has a code in $A_{k+1} \backslash A_{k}$, and we let $X$ be the set of all $x_{k}$. We ensure that a function is in $A$ if it computable from $X$, elements in $A$, and some $\mathrm{S}_{k}^{2}$. With this in place, we construct the model $\mathcal{M}$ as in the proof of Theorem 3.5 Secondly, our construction of $X$ shall satisfy the following requirements.

(1) The set $X$ is covered by a computable sequence of $\left(a_{i}, b_{i}\right)$ of disjoint open intervals with a total measure of $\leq \frac{1}{2}$.

(2) The set of cluster-points of $X$ has measure $\geq \frac{1}{4}$.

(3) There is no cluster point of $X$ in $A$ or in any of the intervals $\left(a_{i}, b_{i}\right)$.

We now establish that items (10)-(3) guarantee that WHBC fails in the model $\mathcal{M}$ for $\epsilon<\frac{1}{4}$. Indeed, assume that $\left\{\left(a_{1}, b_{1}\right), \ldots,\left(a_{n}, b_{n}\right),\left(c_{1}, d_{1}\right), \ldots,\left(c_{m}, d_{m}\right)\right\}$ covers $X$ where all $c_{j}$ and $d_{j}$ are in $\mathcal{M}$ and $\varepsilon<\sum_{1 \leq j \leq m}\left(d_{j}-c_{j}\right)$. By item (3), no cluster point of $X$ is contained in an $\left(a_{i}, b_{i}\right)$ or equals a $c_{j}$ or $d_{j}$, for $i \leq n$ or $j \leq m$. It follows that every cluster point of $X$ must be in some $\left(c_{j}, d_{j}\right)$, since it otherwise will have a positive distance to a set containing $X$ as a subset. However, this is in conflict with the choice of $\epsilon<\frac{1}{4}$ and that the measure of the set of cluster points is larger than $\frac{1}{4}$.

Finally, we construct a set $X$ satisfying items (11)-(3). Let $K \subseteq[0,1]$ be homeomorphic to the Cantor set, but of measure $\geq \frac{1}{2}$. It is straightforward to construct a set like this such that the complement is the pairwise disjoint union of a computable sequence $\left\{\left(a_{i}, b_{i}\right)\right\}_{i \in \mathbb{N}}$ of open intervals with rational endpoints. Let $\left\{y_{k}\right\}_{k \in \mathbb{N}}$ be an enumeration of $A \cap K$. We first select closed intervals $I_{i}$ with $y_{i}$ in the interior and with length $<2^{-(i+3)}$, ensuring that the union of these intervals has measure $<\frac{1}{4}$. When selecting $x_{k}$, we make sure that if $x_{k} \in I_{i}$, then $k<i$. This ensures that $y_{i}$ is not a cluster point of the final set $X$.

Next, define $Z$ as the set of $z \in K$ such that $z \notin \bigcup_{i \in \mathbb{N}} I_{i} ; Z$ clearly has measure at least $\frac{1}{4}$. We shall now define $X$ in such a way that all elements of $Z$ are cluster points of $X$ and that all cluster points of $X$ are in $K$. To this end, let $\left\{z_{j}: j \in \mathbb{N}\right\}$ be a countable and dense subset of $Z$. It suffices to show that all $z_{j}$ are cluster-points of $X$ and that all cluster-points of $X$ are in $K$.

As noted above, $X$ is a sequence $\left\{x_{k}\right\}_{k \in \mathbb{N}}$, and we now define the latter. To define $x_{k}$ for $k=\langle j, n\rangle$, we note that any open interval containing $z_{j}$ will intersect $[0,1] \backslash K$, since $K$ contains no open intervals. Thus, we can find $y$ such that $y \notin K$, $y \in A_{k+1} \backslash A_{k}$, the distance from $y$ and $z_{j}$ is less than $2^{-k}$, and $y \notin I_{i}$ for $i \leq k$. Define $x_{k}$ as this $y$ and note that the resulting set $X$ satisfies item (11)-(13).

One can obtain similar results for other theorems related to the RM of WWKL. For instance, the $\mathrm{L}_{2}$-statement every closed set of positive measure contains a perfect 
sub-set is intermediate between $\mathrm{WKL}_{0}$ and $\mathrm{WWKL}_{0}([59])$. The generalisation of this statement involving Definition 1.2 should imply WHBC. Moreover, the conclusion of the latter essentially expresses that the measure of $[0,1] \backslash \cup_{n \in \mathbb{N}}\left(a_{n}, b_{n}\right)$ is 0 ; one could strengthen this to Borel's notion 'strongly measure zero' ([6]). One could also study 'probabilistic choice', which for $2^{\mathbb{N}}$ and $[0,1]$ is equivalent to WWKL in the Weihrauch lattice (see 3]). For closed sets as in Definition 1.2 realisers for this choice principle would exhibit behaviour similar to $\beta^{3}$ as in Theorem 3.10

We also have the following, based on a consequence of the Lindelöf lemma.

Corollary 3.13. The system $Z_{2}^{\omega}$ cannot prove the following statement: a locally bounded function on $\mathbb{R}$ is dominated by a continuous function on $\mathbb{R}$.

Proof. Note that the statement from the corollary implies item (国) from Theorem 3.8, when combined with WKL. Theorem 3.5 finishes the proof.

3.4. Heine-Borel theorem for non-basic coverings. We study the Heine-Borel theorem for countable coverings consisting of arbitrary open sets. These results are both complimentary (see Theorem 3.15) and establish the robustness of the previous results (see Theorem 3.16).

First of all, we define a 'strong' version of HBC as follows.

Definition 3.14. $\left[\mathrm{HBC}_{\mathrm{s}}\right]$ Let $C \subseteq[0,1]$ be closed and let $\left(O_{n}\right)_{n \in \mathbb{N}}$ be a sequence of open sets with $C \subseteq \cup_{n \in \mathbb{N}} O_{n}$. Then $C \subseteq \cup_{n \leq n_{0}} O_{n}$ for some $n_{0} \in \mathbb{N}$.

We note that coverings by open sets (rather than just intervals) are also studied in e.g. [50, IV.1.5] and [8, Ch. III]. Also note that adding the complement of $C$ to the covering, we get a covering of $[0,1]$, so we may as well restrict the definition to $C=$ $[0,1]$. The strong version $\mathrm{HBC}_{\mathrm{s}}$ is not provable from third-order comprehension.

Theorem 3.15. The system $\mathrm{Z}_{2}^{\omega}$ cannot prove $\mathrm{HBC}_{\mathrm{s}}$ for $C=[0,1]$.

Proof. By Theorem 3.5 since $\mathrm{HBC}$ is a special case of $\mathrm{HBC}_{\mathrm{s}}$ for $C=[0,1]$.

Despite its larger scope, $\mathrm{HBC}_{\mathrm{s}}$ is not that different from $\mathrm{HBC}$, as follows.

Theorem 3.16. The system $\mathrm{RCA}_{0}^{\omega}+\mathrm{QF}-\mathrm{AC}^{0,1}$ proves $\mathrm{WKL} \leftrightarrow \mathrm{HBC}_{\mathrm{s}}$.

Proof. The reverse direction follows from Corollary 3.4 The forward implication follows from Theorem 3.3 with slight modification as follows. In case $\left(\exists^{2}\right)$, one uses the same 'proof by contradiction' as in the proof of Theorem 3.3. starting with $\left(\forall m^{0}\right)(\exists x \in C)\left[x \notin \cup_{n \leq m} O_{n}\right]$ and using $\exists^{2}$ to decide $x \in O_{n}$ whenever necessary. In case $\neg\left(\exists^{2}\right)$, we consider [26, Prop. 4.4 and 4.10]. These theorems establishes that in $\mathrm{RCA}_{0}^{\omega}+\mathrm{WKL}$, one can prove that a function $Y^{2}$ that is continuous on $2^{\mathrm{N}}$, also has an RM-code on $2^{\mathbb{N}}$. Careful inspection of the proofs shows that (i) it also applies to other compact spaces like $[0,1]$ and (ii) one can also prove that a sequence of continuous functions has a sequence of RM-codes. Hence, we may assume $O_{n}$ is given by an RM-code $\alpha_{n}$ for a continuous function on [0,1]. By [50, II.7.1], such an $\alpha_{n}$ (effectively) gives rise to RM-codes for open sets. In this way, $\mathrm{HBC}_{\mathrm{s}}$ reduces to its second-order version, and the latter is provable in $\mathrm{WKL}_{0}$ by [50, IV.1.5].

\section{CODING OPEn SETS}

We study the questions raised in Section 1.2 concerning representations of open sets. In Section 4.1, we study the coding principle open expressing that every characteristic function of an open set has an RM-code. We show that $Z_{2}^{\Omega}$ proves open, 
while $Z_{2}^{\omega}$ cannot. We also show that $\Pi_{k}^{1}-\mathrm{CA}_{0}^{\omega}+$ open proves (second-order) transfinite recursion for $\Pi_{k}^{1}$-formulas, where the latter is intermediate between $\Pi_{k}^{1}-C A_{0}$ and $\Pi_{k+1}^{1}-\mathrm{CA}_{0}$. We show that various theorems pertaining to open sets, like the Cantor-Bendixson or perfect set theorem, imply open or the associated comprehension principle BOOT $^{-}$. In Section 4.2, we study how hard it is to compute a code as in open in terms of the other data. We show that no type two functional suffices, while $\exists^{3}$ does; the latter however yields full $Z_{2}$. In Section 7 , we shall study similar results for finer representations (compared to Definition 1.2).

4.1. Reverse Mathematics. We study the following theorem pertaining to the countable representation of open sets. Let $\left(q_{n}, r_{n}\right)_{n \in \mathbb{N}}$ be a fixed enumeration of all non-trivial open balls $B\left(q_{n}, r_{n}\right)$ with rational center and radius. Note that we use 'open set' in the sense of Definition 1.2 .

Definition 4.1. [open] For every open set $Y \subseteq \mathbb{R}$, there is $X \subset \mathbb{N}$ such that $(\forall n \in \mathbb{N})\left(n \in X \leftrightarrow B\left(q_{n}, r_{n}\right) \subseteq Y\right)$.

Note that for $X, Y$ as in open, we have $x \in Y \leftrightarrow(\exists m \in \mathbb{N})(m \in X \wedge x \in$ $\left.B\left(q_{m}, r_{m}\right)\right)$, i.e. open endows open sets in $\mathbb{R}$ with a countable representation. According to Bourbaki ([7, p. 222]), Cantor first proved that open sets can be written as countable unions of open intervals, i.e. open also carries some historical interest. However, Aczel states in [1, p. 134] that constructive set theory cannot prove open.

We need the following comprehension principle, which is a special case of the comprehension principle BOOT from Section 2.2 and is inspired by [50, V.5.2].

Definition 4.2. BOOT $^{-}$] For $Y^{2}$ such that $(\forall n \in \mathbb{N})\left(\exists\right.$ at most one $\left.f^{1}\right)(Y(f, n)=$ $0)$, we have $(\exists X \subset \mathbb{N})(\forall n \in \mathbb{N})\left(n \in X \leftrightarrow\left(\exists f^{1}\right)(Y(f, n)=0)\right)$.

The name of the previous principle is derived from the verb 'to bootstrap', as combining the relatively weak 4 (in isolation) principles $\left(\exists^{2}\right)$ and $\mathrm{BOOT}^{-}$, gives rise to the much stronger principle of arithmetical transfinite recursion, as in Theorem 4.3. A more general result is proved in Corollary 4.6 below.

Theorem 4.3. The system $\mathrm{ACA}_{0}^{\omega}+\mathrm{BOOT}^{-}$implies $\mathrm{ATR}_{0}$.

Proof. We recall [50, V.5.2] which states that $\mathrm{ATR}_{0}$ is equivalent to the following second-order version of $\mathrm{BOOT}^{-}$: for every arithmetical $\varphi(n, X)$ such that $(\forall n \in$ $\mathbb{N})(\exists$ at most one $X \subseteq \mathbb{N}) \varphi(n, X)$, there is $Z \subset \mathbb{N}$ such that

$$
(\forall n \in \mathbb{N})(n \in Z \leftrightarrow(\exists X \subset \mathbb{N}) \varphi(n, X)) .
$$

To prove the latter principle, we consider the Kleene normal form lemma as in [50, V.5.4] which expresses that an arithmetical formula $\psi(X)$ is equivalent to $\left(\exists f^{1}\right)\left(\forall n^{0}\right) \theta_{0}(\bar{X} n, \bar{f} n)$, where the formula $\theta_{0}$ is bounded and we also have that $(\forall X)\left(\exists\right.$ at most one $\left.f^{1}\right)\left(\forall n^{0}\right) \theta_{0}(\bar{X} n, \bar{f} n)$. In this light, for arithmetical $\varphi(n, X)$, we have that $(\forall n \in \mathbb{N})\left(\exists\right.$ at most one $\left.X^{1}\right) \varphi(n, X)$ is equivalent to the formula $(\forall n \in$ $\mathbb{N})\left(\exists\right.$ at most one $\left.f^{1}\right)(Y(f, n)=0)$ for some $Y^{2}$ defined in terms of $\exists^{2}$. Applying $\mathrm{BOOT}^{-}$then yields the required set $Z$ as in (4.1).

The importance of BOOT $^{-}$is illustrated by Theorem 4.5. We also need the following principle from [57, which is used in [35, §3] to derive e.g. HBU.

\footnotetext{
${ }^{4}$ Note that $\mathrm{ACA}_{0}^{\omega}$ is conservative over $\mathrm{ACA}_{0}$ by [23, Cor. 2.5], while the ECF-translation of BOOT is provable in $A C A_{0}$, i.e. RCA $A_{0}^{\omega}+$ BOOT has the first-order strength of $A_{C A}$.
} 
Definition 4.4. [NFP] For any $\Pi_{\infty}^{1}$-formula $A$ with any parameter:

$$
\left(\forall f^{1}\right)\left(\exists n^{0}\right) A(\bar{f} n) \rightarrow\left(\exists \gamma^{1} \in K_{0}\right)\left(\forall f^{1}\right) A(\bar{f} \gamma(f)) .
$$

Here, ' $\gamma^{1} \in K_{0}$ ' expresses that $\gamma^{1}$ is an associate, which is the same as a code from RM by [26, Prop. 4.4]. Formally, ' $\gamma^{1} \in K_{0}$ ' is the following formula:

$$
\left(\forall f^{1}\right)\left(\exists n^{0}\right)\left(\gamma(\bar{f} n)>_{0} 0\right) \wedge\left(\forall n^{0}, m^{0}, f^{1},\right)\left(m>n \wedge \gamma(\bar{f} n)>0 \rightarrow \gamma(\bar{f} n)={ }_{0} \gamma(\bar{f} m)\right) .
$$

The value $\gamma(f)$ for $\gamma \in K_{0}$ is defined as the unique $\gamma(\bar{f} n)-1$ for $n$ large enough.

We now have the following theorem, where BOOT was introduced in Section 2.2 .

Theorem 4.5. The system $\mathrm{RCA}_{0}^{\omega}$ proves $\mathrm{BOOT} \rightarrow\left[\right.$ open $\left.+\mathrm{ACA}_{0}\right] \rightarrow \mathrm{BOOT}^{-}$and $\mathrm{RCA}_{0}^{\omega}+\mathrm{IND}^{\omega}$ proves $\mathrm{NFP} \rightarrow \mathrm{BOOT} \rightarrow \mathrm{HBU}_{\text {closed }}$.

Proof. The implication NFP $\rightarrow$ BOOT follows from the proof of [45, Theorem 3.5] combined with [46, Theorem 3.6]. A sketch is as follows: assume that BOOT is false for some $Y_{0}$. The formula expressing this has the form $(\forall X \subset \mathbb{N})(\exists n \in \mathbb{N}) A(X, n)$, and a trivial modification yields $(\forall X \subset \mathbb{N})(\exists n \in \mathbb{N}) B(\bar{X} n)$, i.e. only the first $n$ digits of $X$ are used. Let $\gamma \in K_{0}$ be as provided by NFP and use WKL to obtain an upper bound on $C$ on $\gamma$. However, IND ${ }^{\omega}$ proves 'bounded comprehension' (see [50, II.3.9]) for arbitrary formulas, yielding a contradiction.

For [open $\left.+\mathrm{ACA}_{0}\right] \rightarrow \mathrm{BOOT}^{-}$, in case $\neg\left(\exists^{2}\right)$, all functions on Baire space are continuous by [27, §3]. Thus, $\left(\exists f^{1}\right)(Y(f, n)=0)$ is equivalent to $\left(\exists \sigma^{0^{*}}\right)(Y(\sigma *$ $00 \ldots, n)=0)$, and $\mathrm{ACA}_{0}$ provides the set required by $\mathrm{BOOT}^{-}$. In case $\left(\exists^{2}\right)$, let $Y^{2}$ satisfy $(\forall n \in \mathbb{N})\left(\exists\right.$ at most one $\left.f^{1}\right)(Y(f, n)=0)$. The formula $\left(\exists f^{1}\right)(Y(f, n)=0)$ is equivalent to $\left(\exists X \subset \mathbb{N}^{2}\right)(Y(F(X), n)=0)$, where $F(X)(n):=(\mu m)((n, m) \in X)$. Hence, $\left(\exists f^{1}\right)(Y(f, n)=0)$ is equivalent to a formula $\left(\exists f \in 2^{\mathbb{N}}\right)(\tilde{Y}(f, n)=0)$, where $\tilde{Y}$ is defined explicitly in terms of $Y$ and $\mu^{2}$. Now define $Z: \mathbb{R} \rightarrow \mathbb{R}$ as:

$$
Z(x):=\left\{\begin{array}{ll}
0 & n<_{\mathbb{R}}|x| \leq_{\mathbb{R}} n+1 \wedge \tilde{Y}(\eta(x)(0), n) \times \tilde{Y}(\eta(x)(1), n)=0 \\
1 & \text { otherwise }
\end{array},\right.
$$

where $\eta(x)$ provides a pair consisting of the binary expansions of $x-\lfloor x\rfloor$; the pair consists of identical elements if there is a unique such expansion. Note that $\exists^{2}$ can define such functionals $Z$ and $\eta^{1 \rightarrow(1 \times 1)}$. By definition, for each $n \in \mathbb{N}$ there is at most one real $y \in(n, n+1]$ such that $Z(y)=0$. Hence, $Z$ is open and we may apply open to obtain $X \subset \mathbb{N}$ such that $(\forall n \in \mathbb{N})\left(n \in X \leftrightarrow B\left(q_{n}, r_{n}\right) \subset Z\right)$. Now note that for any $m \in \mathbb{N}$, the left-hand side of the following formula is decidable:

$$
\bar{B}\left(m+\frac{1}{2}, \frac{1}{2}\right) \subset Z \leftrightarrow\left(\forall f^{1}\right)(Y(f, m)>0),
$$

which is sufficient to obtain $\mathrm{BOOT}^{-}$in this case. The law of excluded middle now finishes this part of the proof. For the implication BOOT $\rightarrow$ open, we use $\left(\exists^{2}\right) \vee \neg\left(\exists^{2}\right)$ as follows: in the former case BOOT readily yields the set $X$ as in open, while $A C A_{0}$ does the job in the latter case as quantifiers over the reals may be replaced by quantifiers over the rationals if all functions on $\mathbb{R}$ are continuous (as they are in this case by [27, §3]).

For the final implication, BOOT $\rightarrow$ open means that $\mathrm{HBC}$ reduces to $\mathrm{HBC}_{\mathrm{rm}}$, and the latter is equivalent to $\mathrm{WKL}_{0}$ by [8, Lemma 3.13]. We therefore have access to $\mathrm{HBC}$, as well as $\left(\exists^{2}\right)$ in the same way as in the previous paragraphs. Now let $C \subseteq[0,1]$ be closed and fix $\Psi: I \rightarrow \mathbb{R}^{+}$. Use BOOT to define $\left(a_{n}, b_{n}\right)$ as $B\left(q_{n}, r_{n}\right)$ in case $(\exists x \in C)\left(x \in B\left(q_{n}, r_{n}\right) \subseteq I_{x}^{\Psi}\right)$, and $\emptyset$ otherwise. Then clearly $\cup_{n \in \mathbb{N}}\left(a_{n}, b_{n}\right)$ 
covers $C$, and HBC yields a finite sub-covering. Now use $\mathbf{I N D}^{\omega}$ to conclude that this finite sub-covering yields a finite sub-covering for $\cup_{x \in C} I_{x}^{\Psi}$ 'by definition'.

In the following corollary, the second-order system $\Pi_{k}^{1}-\mathrm{TR}_{0}$ is transfinite recursion for $\Pi_{k}^{1}$-formulas, which lies between $\Pi_{k}^{1}-\mathrm{CA}_{0}$ and $\Pi_{k+1}^{1}-\mathrm{CA}_{0}$ (see [50, Table 4]).

Corollary 4.6. The system $\Pi_{k}^{1}-\mathrm{CA}_{0}^{\omega}+$ open implies $\Pi_{k}^{1}-\mathrm{TR}_{0}$.

Proof. The case $k=0$ follows from the theorem and Theorem 4.3. The general case follows in the same way: one simply observes that the proof of ' $2 \rightarrow 1$ ' from [50, V.5.2] relativises to $\mathrm{S}_{k}^{2}$ for $k^{0}>0$.

Thus, we have established that open is hard to prove and moreover is 'explosive' as it becomes much stronger when combined with (higher-order) comprehension.

Finally, we show that BOOT ${ }^{-}$shows up in the study of the Cantor-Bendixson theorem and located sets. Now, the former theorem states that a closed set can be expressed as the union of a perfect closed set and a countable set; this theorem for RM-closed sets is equivalent to $\Pi_{1}^{1}-\mathrm{CA}_{0}$ ([50, VI.1.6]).

Principle 4.7 (CBT). For any closed set $C \subseteq \mathbb{R}$, there exist $P, S \subset C$ such that $C=P \cup S, P$ is perfect and closed, and $S^{0 \rightarrow 1}$ is a sequence of reals.

To be absolutely clear, the countable set $S$ is given as a sequence of real numbers, just like in second-order RM. Furthermore, a set $C$ in a metric space is located if $d(x, C)=\inf _{y \in C} d(x, y)$ exists as a continuous function. By [19, Theorem 1.2], $\mathrm{ACA}_{0}$ is equivalent to the locatedness of non-empty RM-closed sets in the unit interval. Similar results hold for the suprema of RM-closed sets.

Principle 4.8 (CLO). Any non-empty closed set $C \subseteq \mathbb{R}$ is located.

By [26, §4], a continuous function on Baire space (and the same for $\mathbb{R}$ ) has a $\mathrm{RM}$-code in $\mathrm{RCA}_{0}^{\omega}+\mathrm{ACA}_{0}$, i.e. the notion of continuity in CLO does not matter.

Let QF-AC! $C^{0,1}$ be QF-AC ${ }^{0,1}$ restricted to $Y$ such that $\left(\forall n^{0}\right)\left(\exists ! f^{1}\right)(Y(f, n)=0)$, i.e. unique existence. We have the following theorem.

Theorem $4.9\left(\mathrm{RCA}_{0}^{\omega}\right)$. Either $\mathrm{CLO}$ or CBT implies $\mathrm{BOOT}^{-}+\mathrm{QF}_{-} \mathrm{AC}_{!}^{0,1}$.

Proof. The proof is trivial in case $\neg\left(\exists^{2}\right)$, in the same way as in the proof of Theorem 4.5. Thus, we may assume $\left(\exists^{2}\right)$. Let $Y^{2}$ be as in BOOT ${ }^{-}$and let $C$ be the complement of the open set $Z$ defined in (4.2) in the proof of Theorem 4.5, By definition, for each $n \in \mathbb{N}$ there is at most one real $y \in(n, n+1]$ such that $Z(y)=0$. To obtain $\mathrm{BOOT}^{-}$, we proceed as follows.

First assume CLO and note that to check whether $\left(\exists f^{1}\right)(Y(f, n)=0)$, it suffices to: (i) check $Z\left(n+\frac{1}{2}\right)=0$, (ii) if $Z\left(n+\frac{1}{2}\right) \neq 0$, then consider $d\left(n+\frac{1}{2}, C\right)$; the latter is inside $(n, n+1]$ if and only if $\left(\exists f^{1}\right)(Y(f, n)=0)$. Inequalities of real numbers can be decided by $\exists^{2}$, and $\mathrm{BOOT}^{-}$readily follows.

Secondly, assume CBT and note that the set $C$ by assumption consists of isolated points (only). Also, $\left(\exists f^{1}\right)(Y(f, n)=0)$ is equivalent to a point of $C$ being in $(n, n+1]$. Now, for the sets $P, S$ provided by CBT, we must have $P=\emptyset$ and $S$ lists all points of $C$. Thus, BOOT ${ }^{-}$readily follows using $\exists^{2}$, and we are done.

Thirdly, to prove QF-AC! , $^{0,1}$, assume $\left(\forall n^{0}\right)\left(\exists ! f^{1}\right)(Y(f, n)=0)$ and again consider the aforementioned set $C$. By assumption, for every $n$ there is exactly one $y \in \mathbb{R}$ 
such that $y \in(n, n+1] \cap C$. In the same way as in the previous two paragraphs, the set $S$ from CBT and the function $d(x, C)$ from CLO allow one to find this unique real. The theorem now follows.

As a corollary, we show that the perfect set theorem for our notion of closed set as in Definition 1.2 also gives rise to BOOT $^{-}$. The second-order version of the perfect set theorem is equivalent to $\mathrm{ATR}_{0}$ by [50, V.5.5]. Note that a set is uncountable in $\mathrm{RM}$ if there is no sequence that lists its elements (see e.g. [50, p. 193]).

Principle 4.10 (PST). For any closed and uncountable set $C \subseteq[0,1]$, there exist $P \subseteq C$ such that $P$ is perfect and closed.

Corollary 4.11. The system $\mathrm{RCA}_{0}^{\omega}$ proves $\mathrm{PST} \rightarrow \mathrm{BOOT}^{-}$.

Proof. The set $C$ from the proof of the theorem does not have perfect subsets, and hence PST provides a sequence that lists the elements of $C$. The proof of the theorem now yields BOOT $^{-}$.

We have shown that the Cantor-Bendixson theorem, the perfect set theorem, and located sets give rise to BOOT $^{-}$when using closed sets as in Definition 1.2. Hence, a slight change to the aforementioned theorems makes them much harder to prove by the above. Similar results no doubt exist for other theorems pertaining to closed sets from RM. We do not know whether BOOT ${ }^{-}$gives rise to (nice) equivalences, but do have Theorem 4.12 to offer in this regard.

Theorem 4.12. Over $\mathrm{RCA}_{0}^{\omega}$, we have the following results.

(a) $\left(\exists^{3}\right) \rightarrow \mathrm{CLO} \rightarrow \mathrm{HBC}$.

(b) $\mathrm{CLO} \leftrightarrow\left[\mathrm{ACA}_{0}+\right.$ open $]$.

Proof. Item (a) is immediate from item (b) and the usual interval halving technique using $\exists^{3}$; alternatively, note that ' $x \in C$ ' can be replaced by $d(x, C)=_{\mathbb{R}} 0$ and use the proof of Theorem 3.3 with QF-AC ${ }^{0,1}$ omitted. For the reverse direction in item (b), 19, Theorem 1.2] implies that $\mathrm{ACA}_{0}$ is equivalent to the locatedness of nonempty RM-closed sets in the unit interval. This also establishes CLO $\rightarrow \mathrm{ACA}_{0}$ as the antecedent applies to RM-closed sets, while a code for $d(x, C)$ is not needed in the proof. For the forward direction in item (b) , continuous functions on $\mathbb{N}^{\mathbb{N}}$ (and similar for $\mathbb{R}$ ) have RM-codes given $\mathrm{ACA}_{0}$ by [26, §4]. Hence, for an open set $O$, we have $x \in O \leftrightarrow d\left(x, O^{\mathrm{c}}\right)>_{\mathbb{R}} 0$ where the latter is a code for the distance function provided by CLO. However, the right-hand side of this equivalence defines an RM-open set by [50, II.7.1], i.e. open follows immediately.

The notion of weakly located set, studied in e.g. [19], gives rise to a version of item (b) at the level of $\mathrm{WKL}_{0}$. We can obtain the same results as in Theorem 4.12 for CLO replaced by 'for a sequence of closed sets $C_{n} \subset[0,1]$, there is a sequence $\left(x_{n}\right)_{n \in \mathbb{N}}$ such that $\sup C_{n}=x_{n}$ '. We can also establish results like $\left[\mathrm{ACA}_{0}+\mathrm{CBT}^{\prime}\right] \leftrightarrow$ $\left[\Pi_{1}^{1}-\mathrm{CA}_{0}+\right.$ open] over $\mathrm{RCA}_{0}^{\omega}$, where $\mathrm{CBT}^{\prime}$ is CBT with the 'extra data' that $P$ is located in $C=P \cup S$. The same holds for $\mathrm{PST}^{\prime} \leftrightarrow\left[\mathrm{ATR}_{0}+\right.$ open] while the extra 'locatedness' data does seem necessary, i.e. no equivalence is possible without.

The point of the previous results is twofold: on one hand, Theorem 4.12 shows that locating closed sets as in Definition 1.2 is hard, while this operation can be done without the Axiom of Choice. On the other hand, the previous equivalences reduce to known second-order results under ECF from Remark 2.4, as open sets 
as in Definition 1.2 are translated to open sets as in RM by [50, II.7.1]. In other words, second-order RM can be seen as a reflection of higher-order RM under a lossy translation. This observation constitutes the main motivation for the results in [46], where the comparison is made with Plato's ideas on ideal objects.

Finally, a different notion of open set, namely given by uncountable unions of open balls, yields nice equivalences involving BOOT, Cantor-Bendixson theorem, perfect set theorem, and located sets, as explored in [46]. Moreover, the results pertaining to CBT and PST are nice, but seem to depend on the particular notion of '(un)countable' used. As also shown in [46], this problem does not occur for open sets given by uncountable unions of open balls, i.e. the usual definition of 'countable' can be used.

4.2. Computability theory. In this section, we study the computational properties of open, i.e. how hard is it to compute (Kleene S1-S9) the representation provided by this coding principle? Analogous results are obtained in Section 7 for 'finer' representations of open sets.

First of all, we introduce two functionals based on Definition 1.2 as follows. As for $\Theta$-functionals, we simplify the associated type to ' 3 '.

Definition 4.13. [Open sets and computability]

(1) A $\Phi$-functional is any $\Phi^{3}$ such that for every open set $Y \subset \mathbb{R}$ and real $x \in Y$, $\Phi(Y, x)$ equals a rational $r^{0}>0$ such that $B(x, r) \subset Y$.

(2) A $\Psi$-functional is any $\Psi^{3}$ that for every open set $Y \subset \mathbb{R}, \Psi(Y)$ equals two sequences of rationals $\left(a_{n}\right)_{n \in \mathbb{N}},\left(r_{n}\right)_{n \in \mathbb{N}}$ such that $Y=\cup_{n \in \mathbb{N}} B\left(a_{n}, r_{n}\right)$.

It goes without saying that these functionals are not unique. We allow $r_{n}=0$ and note that those instances can be removed using $\mu^{2}$ in case $Y \neq \emptyset$. Note that one can test for the latter condition for open sets using $\mu^{2}$.

Note that $\Phi$-functionals are computable in $\mu^{2}$ and $\exists^{3}$, while the same holds for $\Psi$-functionals by Theorem 4.17, a better result cannot be expected by the following.

Theorem 4.14. No $\Phi$-functional is countably based.

Proof. Assume that $\Phi(Y, 0)=r$, where $0 \in Y$, and where $Y$ is the constant 1, i.e. it represents $\mathbb{R}$. If $\Phi$ is countably based, there is a countable set $X$ such that if $Y^{\prime}$ agrees with $Y$ on $X$ and defines an open set, then $\Phi(Y, 0)=\Phi\left(Y^{\prime}, 0\right)$. However, there will be a real $r^{\prime} \notin X$ such that $0<r^{\prime}<r$. Then consider $Y^{\prime}:=Y \backslash\left\{r^{\prime}\right\}$. Since $r$ is an unacceptable value for $\Phi\left(Y^{\prime}, 0\right)$, we obtain a contradiction.

Corollary 4.15. No $\Psi$-functional is countably based.

Proof. Note that any $\Psi$-functional computes a $\Phi$-functional modulo $\mu^{2}$ : the latter can be used to find the right open ball in the sequence provided by $\Psi$. The class of countably based functionals is closed under Kleene computability (see [20]).

Recall from Section 2.2 that realisers for $\mathrm{HBU}_{\mathrm{c}}$ are called $\Theta$-functionals and compute the finite sub-covering in $\mathrm{HBU}_{\mathrm{c}}$ in terms of the other data. We similarly use $\Theta_{\text {closed }}$ to denote a realiser for $\mathrm{HBU}_{\text {closed }}$ from Section 3 ,

Theorem 4.16. A realiser $\Theta_{\text {closed }}$ for $\mathrm{HBU}_{\text {closed }}$ together with $\mu^{2}$ computes a $\Psi$ functional. 
Proof. Fix some open set $U \subset[0,1]$ and let $C$ be its complement in $[0,1]$. Define $\Psi_{n}(x)$ as $\frac{1}{2^{n}}$ if $x \in C$, and 0 otherwise. Clearly, for fixed $n^{0}, \lambda x . \Psi_{n}(x)$ yields a covering of $C$, implying

$$
\left(\forall n^{0}\right)\left(\exists y_{1}, \ldots, y_{k} \in C\right)(\forall x \in C)\left(x \in \cup_{i \leq k} I_{y_{i}}^{\Psi_{n}}\right),
$$

where $w_{n}:=\Theta_{\text {closed }}\left(\lambda x . \Psi_{n}(x)\right)$ provides the finite sequence of $y_{i}$ 's. With minor modification, $[0,1] \backslash \cup_{n \in \mathbb{N}}\left(\cup_{i<\left|w_{n}\right|} I_{w_{n}(i)}^{\Psi_{n}}\right)$ yields a countable union of open intervals $\left(a_{n}, b_{n}\right)$ such that $U=\cup_{n \in \mathbb{N}}\left(a_{n}, b_{n}\right)$.

Finally, the 'standard' proof that an open set in $\mathbb{R}$ is the union of countable many open balls, goes through modulo $\left(\exists^{3}\right)$.

Theorem 4.17. The system $\mathrm{Z}_{2}^{\Omega}$ proves open. A $\Psi$-functional can be computed from $\exists^{3}$ via a term from Gödel's $T$.

Proof. Let $Y \subset \mathbb{R}$ be open and define for $x \in Y$ the (non-empty by definition) sets $A_{x}:=\{a \in \mathbb{R}:(a, x] \subset Y\}$ and $B_{y}:=\{b \in \mathbb{R}:[b, y) \subset Y\}$ using $\exists^{3}$, letting them be the empty set if $x \notin Y$. If the set $A_{x}$ (resp. $B_{x}$ ) has a lower (resp. upper) bound (which is decidable assuming $\exists^{3}$ ), then $a_{x}:=\inf A_{x}$ (resp. $b_{x}:=\sup B_{x}$ ) exists thanks to $\exists^{3}$, using the usual interval-halving technique. In case such a bound is missing, we use a default value for $a_{x}$ (resp. $b_{x}$ ) meant to represent $-\infty$ (resp. $+\infty)$. We define $J_{x}:=\left(a_{x}, b_{x}\right)$ and will show that $Y=\cup_{q \in \mathbb{Q}} J_{q}$, thus establishing the theorem. By the definition of $J_{x}$, we must have $J_{x} \subset Y$ for all $x \in Y$. Since also $Y \subset \cup_{x \in Y} J_{x}$ due to $x \in J_{x}$ if $x \in Y$, we actually have $Y=\cup_{x \in Y} J_{x}$, and note that $\exists^{3}$ guarantees that this union actually exists. One readily shows that either $J_{x}=J_{y}$ or $J_{x} \cap J_{y}=\emptyset$ for $x, y \in Y$. Hence, for $x \in Y, J_{x}=J_{q}$ for any $q \in J_{x} \cap \mathbb{Q}$, and the theorem follows. Note that the second part follows immediately.

\section{The Urysohn and Tietze theorems}

We establish the results sketched in Section 1.2 for the Urysohn and Tietze theorems; the latter are basic results of topology that are well-known in RM: for RMcodes of closed sets, these theorems even hold recursively, i.e. the objects claimed to exists may be computed (in the sense of Turing) from the data by [50, II.7]. We now show that the situation is dramatically different for our notion of closed sets. In particular, we connect these theorems to the coding principle open from the previous section and establish they suffer from the Pincherle phenomenon.

First of all, we study the Urysohn lemma for $\mathbb{R}$ and Definition 1.2 as follows.

Definition 5.1. [URY] For closed disjoint sets $C_{0}, C_{1} \subseteq \mathbb{R}$, there is a continuous function $g: \mathbb{R} \rightarrow[0,1]$ such that $x \in C_{i} \leftrightarrow g(x)=i$ for any $x \in \mathbb{R}$ and $i \in\{0,1\}$.

Definition 5.2. A realiser for URY is called a $\zeta$-functional, i.e. for disjoint closed sets $C_{0}, C_{1} \subset \mathbb{R}, \zeta\left(C_{0}, C_{1}\right)$ is a continuous function such that $\zeta\left(C_{0}, C_{1}\right)(x)=i$ whenever $x \in C_{i}$ for $i=0,1$.

In case one works with codes rather than actual sets, the Urysohn lemma is 'recursively true' by [50, II.7.3], i.e. one can compute (in the sense of Turing) a code for the continuous function from a code for the sets $C_{i}$, over $\mathrm{RCA}_{0}$. How different things are at the higher-order level: we have the following RM and relative computability result. Recall the notion of $\Phi$-functional from Definition 4.13 . 
Theorem 5.3. A $\Phi$-functional can be computed from a $\zeta$-functional and $\mu^{2} . A \zeta$ functional can be computed from a $\Psi$-functional and $\mu^{2} ; \mathrm{ACA}_{0}^{\omega}$ proves $\mathrm{URY} \leftrightarrow$ open.

Proof. For the first part, fix an open set $U \subset \mathbb{R}$, define disjoint closed sets $C_{0}=U^{\mathrm{c}}$ and $C_{1}=\emptyset$, yielding $x \in U \leftrightarrow \zeta\left(U^{\mathrm{c}}, \emptyset\right)(x)>_{\mathbb{R}} 0$ for all $x \in \mathbb{R}$. Since $\lambda x . \zeta\left(U^{\mathrm{c}}, \emptyset\right)$ is continuous, $\mu^{2}$ provides a modulus of (pointwise) continuity by (the proof of) [26. Prop. 4.7]. Using this modulus (and $\mu^{2}$ ), we may find $r>0$ such that for all $y \in B(x, r)$, we have $\zeta\left(U^{\mathrm{c}}, \emptyset\right)(y)>0$, which is exactly as required for a $\Phi$ functional. Note that URY $\rightarrow$ open also follows, over $A_{C A}^{\omega}$. The second part involving $\Psi$-functionals follows by definition.

For the implication open $\rightarrow$ URY over $\mathrm{ACA}_{0}^{\omega}$, define $h(x)$ as $i$ for $x \in C_{i}$ and $i=0,1$. In case $x \in Z=C_{0}^{\mathrm{c}} \cap C_{1}^{\mathrm{c}}$, we define $h(x)$ as follows: first note that $Z$ is open (by definition), and hence $Z=\cup_{n \in \mathbb{N}} B\left(a_{n}, r_{n}\right)$ by open. Note that $Z$ cannot be empty by assumption. Using $\mu^{2}$, we find $m^{0}$ such that $x \in B\left(a_{m}, r_{m}\right)$ and we may test if $a_{m} \pm r_{m}$ belong to $C_{0}$ or $C_{1}$. If such a point is in neither, it is in $Z$, and hence in $B\left(a_{k}, r_{k}\right)$ for some $k \neq m$, and we can repeat the previous process. There are three possible outcomes:

(a) This procedure ends after finitely many steps, say with $a_{k_{0}}-r_{k_{0}}<x<$ $a_{k_{1}}+r_{k_{1}}$ and the former (resp. latter) rational is in $C_{0}$ (resp. $C_{1}$ ).

(b) This procedure only ends after finitely many steps in one direction, say with $a_{k_{0}}-r_{k_{0}}<x$ and this rational is in $C_{0}$.

(c) This procedure never ends in both directions.

If item (드) is the case, then $C_{0}=C_{1}=\emptyset$, and $h(x)=0$ everywhere. If item (国) is the case, define $h(x)$ as the (increasing) straight line connecting $\left(a_{k_{0}}-r_{k_{0}}, 0\right)$ and $\left(a_{k_{1}}+r_{k_{1}}, 1\right)$ for $a_{k_{0}}-r_{k_{0}}<x<a_{k_{1}}+r_{k_{1}}$. If $C_{1}$ (resp. $\left.C_{0}\right)$ is eventually met on the left (resp. right), the modification to $h$ is obvious. If item (b) is the case, then define $h(x):=0$ for $a_{k_{0}}-r_{k_{0}}<x$. If $C_{1}$ is eventually met on the left, or if the unbounded area is on the left, the modification to $h$ is obvious.

Finally, an indirect (but over RCA $A_{0}^{\omega}$ ) proof of open $\rightarrow$ URY follows from replacing the closed sets in the latter by RM-codes and applying the RM-version of the Urysohn lemma, namely [50, II.7.3].

We can weaken the base theory in the previous theorem as follows. Let coco be the statement that every continuous $Y: \mathbb{R} \rightarrow \mathbb{R}$ has an RM-code, as studied in [26, §4] for Baire space. Note that the ECF-interpretation of coco is a tautology.

Corollary 5.4. The system $\mathrm{RCA}_{0}^{\omega}+$ coco proves URY $\leftrightarrow$ open.

Proof. Use $\left(\exists^{2}\right) \vee \neg\left(\exists^{2}\right)$ as follows. In the former case, the theorem provides a proof of URY $\leftrightarrow$ open. In the latter case, all functions on $\mathbb{R}$ are continuous by [27, §3], while coco provides RM-codes. Thus, the usual proof of the second-order Urysohn theorem may be used (see [50, II.7.3]), while open follows from [50, II.7.1].

Secondly, we study the Tietze (extension) theorem, which expresses that a continuous function on a closed set can be extended to a continuous function on the whole space, while if the original function is bounded, so is the extended function with the same bound. Lebesgue $([28])$, de la Vallée-Poussin $([58])$, and Carathéodory ([12]) prove special cases of this theorem not involving boundedness conditions. Furthermore, Tietze states in [55, p. 10] that the given function can be discontinuous outside the closed set and that the boundedness condition may be dropped. We therefore consider the following version of the Tietze theorem. 
Definition 5.5. [TIE] For $f: \mathbb{R} \rightarrow \mathbb{R}$ continuous on the closed $D \subseteq[0,1]$, there is $g: \mathbb{R} \rightarrow \mathbb{R}$, continuous on $[0,1]$ such that $f(x)=\mathbb{R} g(x)$ for $x \in D$.

Theorem 5.6. The system $\mathrm{ACA}_{0}^{\omega}+\mathrm{QF}-\mathrm{AC}^{0,1}$ proves $\mathrm{TIE} \leftrightarrow \mathrm{URY}$ and $\mathrm{RCA}_{0}^{\omega}$ proves $[\mathrm{TIE}+\mathrm{WKL}] \rightarrow \mathrm{HBC}$.

Proof. For the implication URY $\rightarrow$ TIE, we have URY $\rightarrow$ open by Theorem 5.3 and we may therefore use the RM-proof of the Tietze extension theorem (see [50, II.7.5]). The latter applies to bounded functions and we can guarantee boundedness using WKL (using $\mathrm{HBC}_{\mathrm{rm}}$ in particular). Note that $\mathrm{ACA}_{0} \rightarrow$ coco over $R C A_{0}^{\omega}$ is proved in [26, §4], and the same proof goes through relative to closed sets using $\left(\exists^{2}\right)$.

For TIE $\rightarrow$ URY, let $C_{i}$ be as in URY for $i=0,1$ and define $f$ on $C_{2}:=C_{0} \cup C_{1}$ as follows: $f(x)=0$ if $x \in C_{0}$ and 1 otherwise. If $f$ is continuous on $C_{2}$, then its extension $g$ provided by TIE is as required for URY. To show that $f$ is continuous on $C_{2}$, we prove that

$$
\left(\forall N^{0}\right)\left(\exists n^{0}\right)\left(\forall x \in C_{0}, y \in C_{1}\right)\left(x, y \in[-N, N] \rightarrow|x-y| \geq \frac{1}{2^{n}}\right) .
$$

If (5.1) is false, QF-AC ${ }^{0,1}$ yields a double sequence $\lambda n . x_{n}, \lambda n . y_{n}$ in $[-N, N]$ such that for all $n^{0}$, we have $x_{n} \in C_{0}, y_{n} \in C_{1}$, and $\left|x_{n}-y_{n}\right|<\frac{1}{2^{n}}$. As $C_{0}, C_{1}$ are closed and the sequences bounded, there are $x \in C_{0}, y \in C_{1}$ such that $\lim _{n} x_{h_{0}(n)}=x$ and $\lim _{n} y_{h_{1}(n)}=y$ for sub-sequences provided by $h_{0}^{1}, h_{1}^{1}$. However, $\left(\forall n^{0}\right)\left(\left|x_{n}-y_{n}\right|<\right.$ $\frac{1}{2^{n}}$ ) implies that $x=\mathbb{R} y$, a contradiction since $C_{0} \cap C_{1}=\emptyset$. Finally, since (5.1) provides a positive 'distance' between $C_{0}$ and $C_{1}$ in every interval $[-N, N]$, we can always chose a small enough neighbourhood to exclude points from one of the parts of $C$, thus guaranteeing continuity for $f$ everywhere on $C_{2}$.

For $[\mathrm{TIE}+\mathrm{WKL}] \rightarrow \mathrm{HBC}$, let $F$ and $D$ be as in item (d) of Theorem 3.8 and consider the extension $g$ provided by TIE. By WKL, $g$ is bounded on $[0,1]$, and $F$ is therefore bounded on the closed set $D$. Theorem 3.8 finishes this implication.

We improve Theorem 5.6 as follows; the proof is similar to that of Corollary 5.4

Corollary 5.7. The system $\mathrm{RCA}_{0}^{\omega}+\mathrm{QF}-\mathrm{AC}^{0,1}+$ coco proves $\mathrm{TIE} \leftrightarrow \mathrm{URY}$.

In conclusion, we have established that the Urysohn and Tietze theorems suffer from the Pincherle phenomenon. Moreover, the previous corollary does not seem to go through over $\mathrm{RCA}_{0}^{\omega}$, unless we introduce moduli of continuity in the theorems at hand. Another approach would be to extend the base theory; this option is studied in [46, §5] for fragments of NFP from Definition 4.4.

\section{The BAire CATEgory theorem}

We establish the results sketched in Section 1.2 for the Baire category theorem. We introduce realisers for the latter in Section 6.1 and show that these functionals can be computed from $\exists^{2}$ and non-monotone inductive definitions in Section 6.2.

6.1. Introduction. The Baire category theorem expresses that a sequence of dense open sets has an intersection that is also dense; this theorem can be found in Baire's 1899 doctoral thesis and Osgood's paper [39. This theorem is studied (in some variations) in the computational approaches to mathematics mentioned in Remark 1.1 It is therefore a natural question what the computational properties of the Baire category theorem are when using Definition 1.2, Our main results are: 
(a) A realiser $\xi$ for the Baire category theorem (Definition 6.1) can be computed from $\exists^{2}$ and IND, the inductive definition operator (Definition 6.2).

(b) There is no Baire-realiser $\xi$ computable in a functional of type 2 .

Thus, we observe that the Baire category theorem also (partially) exhibits the Pincherele phenomenon. This should be contrasted with the status of the Baire category theorem in e.g. the Weihrauch framework (4 4). We also note that the below proofs are quite different from the proofs in [35, 37. and the above.

Note that by [21, Theorem 4.102], ZF proves that separable completely metrisable spaces have the property of Baire, i.e. the Axiom of Choice is not the cause of the (computational) hardness of the Baire category theorem. The aforementioned notion of realiser is defined as follows.

Definition 6.1. A realiser for the Baire category theorem is any $\xi^{3}$ such that whenever $\left(Y_{n}\right)_{n \in \mathbb{N}}$ is a sequence of dense open sets of reals, then $\xi\left(\lambda n . Y_{n}\right) \in \bigcap_{n \in \mathbb{N}} Y_{n}$.

For the previous definition, we assume a standard coding of the reals in $\mathbb{N}^{\mathbb{N}}$, and that a set $Y \subset \mathbb{R}$ is given in in the form of its characteristic function as in Definition 1.2. So, technically we are working inside the full type-structure over $\mathbb{N}$ up to level 3. All our "algorithms" are relative to $\exists^{2}$ and any other objects specified in the argument.

As noted above, the Baire category theorem for separable spaces can be proved in ZF, yielding the existence of a Baire realiser $\xi$; a direct translation of that argument (however) uses computations relative to $\exists^{3}$. Our first result involves a construction of a Baire realiser that terminates on all countable sequences of sets, and gives an element in the intersection when the sets are all open and dense, working within the realm of the countably based functionals. By contrast, other 'basic' theorems about open sets, like every open set is a union of open intervals with rational endpoints and for every closed set $C$, the distance $d(x, C)$ is continuous, only have realisers that are not countably based.

Definition 6.2. For $\Gamma: 2^{\mathbb{N}} \rightarrow 2^{\mathbb{N}}$ satisfying the monotonicity condition $(\forall A \subseteq$ $\mathbb{N})(\Gamma(A) \supseteq A)$, define the well-ordered sequence of sets $\Gamma_{\alpha}$ as follows:

$$
\Gamma_{\alpha}:=\Gamma\left(\bigcup_{\beta<\alpha} \Gamma_{\beta}\right) \text {. }
$$

For any such $\Gamma$, there is an ordinal $\alpha_{0}$ with $\Gamma_{\alpha_{0}+1}=\Gamma_{\alpha_{0}}$, where the latter is called the inductive closure of $\Gamma$. Finally, for any $F: 2^{\mathbb{N}} \rightarrow 2^{\mathbb{N}}$ we define $\operatorname{IND}(F)$ as the inductive closure $\Gamma_{\alpha_{0}}$ for the functional $\Gamma$ defined as $\Gamma(A):=A \cup F(A)$.

Regarding the previous definition, IND denotes a type three functional, while IND $^{\omega}$ is the induction axiom for all $\mathrm{L}_{\omega}$-functionals.

Finally, we noted above that the Baire category theorem for separable spaces can be proved in ZF and this result will be sharpened, for our special case BCT defined below, as follows.

(c) The system RCA ${ }_{0}^{\omega}+$ BOOT proves the Baire category theorem BCT.

(d) The system $Z_{2}^{\omega}$ cannot prove BCT.

Note that BOOT $\rightarrow$ open by Theorem 4.5 and open sets thus have an RM-representation. However, BCT deals with sequences of open sets and obtaining the associated sequences of RM-representations would seem to require countable choice.

6.2. Main results. We prove the results sketched in items (a)-(d) in Section 6.1. 
6.2.1. Computability Theory. We prove the results sketched in items (a) and (b) in Section 6.1. To this end, we first fix some notation, as follows.

Definition 6.3. [Computational attempts]

- A tag is a pair $(r, \epsilon)$ from $\mathbb{Q}$ where $\epsilon>0$. We let $(r, \epsilon)_{o}=(r-\epsilon, r+\epsilon)$ and $(r, \epsilon)_{c}$ be the corresponding closed interval $[r-\epsilon, r+\epsilon]$.

- If $\left(r_{1}, \epsilon_{1}\right),\left(r_{2}, \epsilon_{2}\right)$ are tags, we let $\left(r_{1}, \epsilon_{1}\right) \prec\left(r_{2}, \epsilon_{2}\right)$ if $r_{1}=r_{2}$ and $\epsilon_{1} \geq 2 \epsilon_{2}$.

- An attempt is a sequence $\mathbf{s}=\left[\left(r_{1}, \epsilon_{1}\right), \ldots,\left(r_{k}, \epsilon_{k}\right)\right]$ of tags, where we for $i<k$ have that $\left(r_{i+1}, \epsilon_{i+1}\right)_{c} \subseteq\left(r_{i}, \epsilon_{i}\right)_{o}$ and that $2 \epsilon_{i+1} \leq \epsilon_{i}$.

- If $\mathbf{s}$ and $\mathbf{t}$ are attempts, we let $\mathbf{s} \triangleleft \mathbf{t}$ if either $\mathbf{s}$ is an initial sub-sequence of $\mathbf{t}$ or if $\mathbf{s}$ comes before $\mathbf{t}$ in the lexicographical ordering on attempts based on ' $\prec$ ' as above for tags.

The ordering ' $\triangleleft$ ' is not the Kleene-Brouwer ordering, but a partial ordering all the same. Intuitively, an attempt will be an attempt to find a shrinking sequence of closed intervals whose single point in the intersection will also be in $\cap_{n \in \mathbb{N}} Y_{n}$. We have limited access to information about any $Y_{n}$ : we do not know if it is open and dense. Even when it is, we still have no way of saying that a tag $(r, \epsilon)$ represents a subset of $Y_{n}$. So certain attempts may lead to failure, and we have to try again with better attempts. This is what will be captured by our inductive definition, which will be defined from a given sequence $\left(Y_{n}\right)_{n \in \mathbb{N}}$ in a uniform way, as follows.

Definition 6.4. Let $\left(Y_{n}\right)_{n \in \mathbb{N}}$ be given, and let $A$ be a set of attempts. We define $\Gamma(A)$ in cases as follows, where we order $\mathbb{Q}$ according to a standard enumeration.

o) If $A$ is not totally ordered by $\triangleleft$, we let $\Gamma(A)=A$. For the rest of the cases we will assume that $A$ is totally ordered.

i) In case $A=\emptyset$ and if $Y_{1} \cap \mathbb{Q}=\emptyset$, put $\Gamma(A)=A$. If not, $\Gamma(A):=\left\{\left(r_{1}, 1\right)\right\}$, where $r_{1}$ is the first rational number in $Y_{1}$.

ii) If the set $A$ has a $\triangleleft$-maximal element $\left[\left(r_{1}, \epsilon_{1}\right), \ldots,\left(r_{k}, \epsilon_{k}\right)\right]$ and if $\left(r_{k}, \epsilon_{k}\right)_{o} \cap$ $Y_{k+1} \cap \mathbb{Q}=\emptyset$ we let $\Gamma(A)=A$. If not, we define

$$
\Gamma(A)=A \cup\left\{\left[\left(r_{1}, \epsilon_{1}\right), \ldots,\left(r_{k}, \epsilon_{k}\right),\left(r_{k+1}, \epsilon_{k+1}\right)\right]\right\},
$$

where $r_{k+1}$ is the first rational number in $\left(r_{k}, \epsilon_{k}\right)_{o} \cap Y_{k+1}$, and then $\epsilon_{k+1}$ is the first positive rational number such that $\left(r_{k+1}, \epsilon_{k+1}\right)_{c} \subseteq\left(r_{k}, \epsilon_{k}\right)_{o}$ and such that $2 \epsilon_{k+1} \leq \epsilon_{k}$

iii) If there is $k \in \mathbb{N}$ with infinitely many attempts $\left[\left(r_{1}, \epsilon_{1}\right), \ldots,\left(r_{k}, \epsilon_{k}\right)\right]$ in $A$, we put $\Gamma(A)=A$.

iv) If none of the above apply, we proceed as follows: for each $k$, let $\left[\left(r_{1}, \epsilon_{1}\right), \ldots,\left(r_{k}, \epsilon_{k}\right)\right]$ be the $\triangleleft$-maximal attempt in $A$ of length $k$. It is easy to see that for $i \leq k$, the tag $\left(r_{i}, \epsilon_{i}\right)$ will be independent of the choice of $k$. Let $x$ be the unique element in $\bigcap_{i \in \mathbb{N}}\left(r, \epsilon_{i}\right)_{c}$. We consider the following sub-cases:

(1) If $x \in \bigcap_{i \in \mathbb{N}} Y_{i}$, let $\Gamma(A)=A$

(2) Otherwise, let $k$ be minimal such that $x \notin Y_{k}$ and we define $\Gamma(A)=$ $A \cup\left\{\left[\left(r_{1}, \epsilon_{1}\right), \ldots,\left(r_{k-1}, \epsilon_{k-1}\right),\left(r_{k}, \frac{\epsilon_{k}}{2}\right)\right]\right\}$.

By construction, in all cases where $A$ is totally ordered by $\triangleleft$, we either define $\Gamma(A)=A$ or we add one new element on top of $A$. This means that $\Gamma$, seen as an inductive definition, will generate a well-ordered set $A_{\infty}$ of attempts such that $\Gamma\left(A_{\infty}\right)=A_{\infty}$. Moreover, we define $\xi\left(\lambda n . Y_{n}\right)=x$ as in case iv).(1) if this is the 'stopping' case. Otherwise, we define $\xi\left(\lambda n . Y_{n}\right)=0$. 
The resulting functional $\xi$ is clearly computable in IND and $\exists^{2}$. It remains to show that iv).(1) will be the 'stopping' case when each $Y_{n}$ is open and dense. We consider the other alternatives, and show that they are impossible in this situation.

- Since we generate a totally ordered set, case o) will never be relevant for any input $\left(Y_{n}\right)_{n \in \mathbb{N}}$.

- Since $Y_{1}$ is open and dense, we start the recursion by adding an element in case i). Since $Y_{k+1}$ is open and dense, we will also continue the recursion when we are in case ii).

- The remaining alternative is case iii). In this case, there will be a least $k$ for which this is possible. Since the only way to develop $A$ sideways (using $\prec$ on tags) is via case iv).2, there will be a maximal attempt $\left[\left(r_{1}, \epsilon_{1}\right), \ldots,\left(r_{k-1}, \epsilon_{k-1}\right)\right]$ of length $k-1$, and tags $\left(r_{k}, \frac{\epsilon_{k}}{2^{n}}\right)$ such that

$$
\left[\left(r_{1}, \epsilon_{1}\right), \ldots,\left(r_{k-1}, \epsilon_{k-1}\right),\left(r_{k}, \frac{\epsilon_{k}}{2^{n}}\right)\right] \in A
$$

for all $n$. By the construction, $r_{k} \in Y_{k}$, and $Y_{k}$ is open, so there is an $n \in \mathbb{N}$ such that $\left(r_{k}, \frac{\epsilon_{k}}{2^{n}}\right)_{c} \subseteq Y_{k}$. But then, whenever we are employing case iv).2 after the attempt $\left[\left(r_{1}, \epsilon_{1}\right), \ldots,\left(r_{k-1}, \epsilon_{k-1}\right),\left(r_{k}, \frac{\epsilon_{k}}{2^{n}}\right)\right]$ enters $A$, we will ask if some $x \in\left(r_{k}, \frac{\epsilon_{k}}{2^{n}}\right)_{c}$ is in the intersection of all $Y_{m}$, and if the answer is that it is not, we will not find $Y_{k}$ to be the guilty one. So, when all $Y_{n}$ are open, the tree of attempts that we are constructing will be finitely branching.

We have now proved the following theorem.

Theorem 6.5. There is a total type 3 functional $\xi$ computable in IND and $\exists^{2}$ such that whenever $\left(Y_{n}\right)_{n \in \mathbb{N}}$ is a sequence of open, dense sets, then $\xi\left(\lambda n . Y_{n}\right) \in \bigcap_{n \in \mathbb{N}} Y_{n}$.

By contrast, we also have the following negative result.

Theorem 6.6. There is no Baire-realiser $\xi$ computable in a functional of type 2.

We will prove this theorem by contradiction. We first have to develop some machinery and associated lemmas; then we will prove the theorem by reference to the machinery and the lemmas.

First of all, assume that there is an index $d$ and a type 2 functional $F$ such that for all sequences $\left(Y_{n}\right)_{n \in \mathbb{N}}$ of subsets of $\mathbb{N}^{\mathbb{N}}$ we have that the following:

(i) the function $f:=\lambda a \in \mathbb{N} .\{d\}\left(F, a,\left(Y_{n}\right)_{n \in \mathbb{N}}\right)$ is total,

(ii) if each $Y_{n}$ is open and dense, then $f \in \bigcap_{n \in \mathbb{N}} Y_{n}$.

We will show that this assumption leads to a contradiction, by constructing, from $d$ and $F$, a sequence $\left(Y_{n}\right)_{n \in \mathbb{N}}$ for which (i) and (ii) fail. The construction is based on Moschovakis' definition of computation trees from 30, but we provide most details. As to notation we write $\mathbf{Y}$ for the sequence $\left(Y_{n}\right)_{n \in \mathbb{N}}$. We also use of a modified version of S8 as in Notation 6.7, which we motivate as follows.

A clear disadvantage of Kleene's original S1-S9 is that the concept is restricted to functionals of pure type. Thus, if we want to compute relative to objects of mixed types, we need to code them within the pure types. Since S8 expresses application of one object to already computed objects of lower types, the coding can make an application of this scheme hard to comprehend. When studying computations relative to specific objects of mixed types at level 2 or 3 , a reformulation of S8 adjusted to a direct application, and not an application of the coded version, makes constructions easier to comprehend. Our modification is equivalent to how S8 would work when all objects were coded into the pure types. 
Notation 6.7 (Complete and incomplete computation).

- A computation tuple is a sequence $\langle e, \vec{a} ; c\rangle$ indicating the terminating computation $\{e\}(F, \mathbf{Y}, \vec{a})=c$, where $\vec{a}$ is a finite sequence of numbers and we modify Kleene's $S 8$ as follows:

- If e $=\langle 8,0, d\rangle$, then $\{e\}(F, \mathbf{Y}, \vec{a}):=F(f)$.

- If $e=\langle 8, n+1, d\rangle$, then

$$
\{e\}(F, \mathbf{Y}, \vec{a}):=\left\{\begin{array}{lll}
0 & \text { if } & f \in Y_{n} \\
1 & \text { if } f \notin Y_{n}
\end{array},\right.
$$

where in both cases $f=\lambda a .\{d\}(F, \mathbf{Y}, a, \vec{a})$.

- In an incomplete computation tuple $\langle e, \vec{a}\rangle$ we leave out the final $c$, indicating that the value of the computation is unknown (possibly forever).

- The set of incomplete computation tuples $\langle e, \vec{a}\rangle$ is enumerated via a standard sequence numbering, and we let $n(\langle e, \vec{a}\rangle)$ be the corresponding number.

Let $\varepsilon$ denote the empty sequence of integers. We assume throughout the construction that $e_{0}=\langle 8,0, d\rangle$ is such that we for all $\mathbf{Y}$ have that $\left\{e_{0}\right\}(F, \mathbf{Y}, \varepsilon) \downarrow$.

Let us first consider the well-understood case where $\mathbf{Y}$ is fixed and $\left\{e_{0}\right\}(F, \mathbf{Y}, \varepsilon) \downarrow$. Then we can find the value $c$ by building the computation tree for the computation by transfinite induction. We start with the top node $\left\langle e_{0}, \varepsilon\right\rangle$, i.e. an incomplete computation tuple, and then in a combined top-down and bottom-up procedure construct a tree of incomplete and complete computation tuples as explained below. In the process, we may add new incomplete computation tuples and we may turn incomplete ones to complete ones. We now give a semi-formal description of the aforementioned inductive process as follows.

- In the case of composition as follows:

$$
\{e\}(F, \mathbf{Y}, \vec{a})=\left\{e_{1}\right\}\left(F, \mathbf{Y},\left\{e_{2}\right\}(F, \mathbf{Y}, \vec{a}), \vec{a}\right)
$$

we first fill in $\left\langle e_{2}, \vec{a}\right\rangle$ as an incomplete sub-computation of $\langle e, \vec{a}\rangle$. When we later observe that $\left\langle e_{2}, \vec{a} ; b\right\rangle$ is the proper sub-computation, we can also fill in $\left\langle e_{1}, b, \vec{a}\right\rangle$ as an incomplete sub-computation. When we then at an even later stage realise that $\left\langle e_{1}, b, \vec{a} ; c\right\rangle$ is the proper sub-computation, we can make $\langle e, \vec{a}\rangle$ complete as $\langle e, \vec{a} ; c\rangle$.

- Primitive recursion can be seen as iterated composition, and is therefore handled in a similar way.

- For the rest of the schemes, it is obvious what is going on: either the incomplete computation tuple at hand is one of an initial computation, and we can fill in the correct value right away, or the set of incomplete tuples for the immediate sub-computations is uniquely given, we have to wait for the process to complete these, and then we can find the right value of the one at hand.

The whole process can be seen as a simultaneous inductive definition of the construction of the tree of incomplete computation tuples and the completion of these.

The above describes the construction when $\mathbf{Y}$ is fixed, but in order to obtain the desired contradiction we will have to construct $\mathbf{Y}$ and the computation tree simultaneously, which adds complications. The major problem is that we do not know $\mathbf{Y}=\left(Y_{n}\right)_{n \in \mathbb{N}}$ when we construct the tree, but we have to make a decision what to answer whenever the procedure for constructing the computation tree requests an answer to $Y_{n}(\lambda a .\{d\}(F, \mathbf{Y}, a, \vec{a}))$. Our solution to this problem is that the first 
time $f$ in the form of $\lambda a .\{d\}(F, \mathbf{Y}, a, \vec{a})$ is needed in our computation tree, as an input to $F$ or to some $Y_{n}$, we define $f \notin Y_{n}$ exactly when $n=n(\langle d, \vec{a}\rangle)$.

One useful feature of this strategy is that $Y_{n}$ then either is all of $\mathbb{N}^{\mathbb{N}}$ or just $\mathbb{N}^{\mathbb{N}}$ with one point missing, so $Y_{n}$ is open and dense. Another useful feature is that $f$ is not an acceptable value of $\xi(\mathbf{Y})$ since $f$ is left out of one $Y_{n}$. One complication is that we have to convert the tree we are constructing into a well-ordering in order to talk about e.g. 'the first occurrence'; another complication is that we have to ensure that whenever we want to give the correct value to an S8-computation tuple, we already know which functions are used in earlier S8-computations.

We will now give the details of the construction. First some conventions and some intuition are needed.

Definition 6.8 (Computation paths).

- A computation path will be a finite sequence $\left(t_{0}, \ldots, t_{k}\right)$ of computation tuples such that $t_{i+1}$ is a sub-computation of $t_{i}$ as defined below. Each $t_{i}$ may be complete or incomplete, but if $t_{i}$ is complete and $j>i$, then $t_{j}$ must also be complete. This reflects that we cannot give a value to a computation without knowing the values of all sub-computations.

- In a complete computation path, all computation tuples are complete.

- If $t$ is a computation tuple, we will order the possible sub-computations. In this ordering, we will not discriminate between an incomplete subcomputation and its possible completion. In the process we are about to define, certain incomplete computation tuples will be turned into complete ones, and we do not want to change the position in the overall ordering.

- When we construct our tree by transfinite recursion, we will refer to the Kleene-Brouwer ordering based on the node-wise ordering of the sub-computations, meaning that if we extend a computation path to a longer one, we move down in the ordering.

Based on Definition 6.8, we now introduce the tree of computation paths. We establish in Lemma 6.11 below that this tree must be well-founded.

Definition 6.9 (Tree of computation paths). By recursion on the ordinal $\alpha$, we construct a tree $T_{\alpha}$ of computation paths as follows.

First of all, if $\alpha=0$, we let $T_{\alpha}$ consist of the single computation-path $\left(t_{0}\right)$, where $t_{0}$ is the incomplete computation tuple $\left\langle e_{0}, \varepsilon\right)$, the computation the process aims to find the value of.

Secondly, if $\alpha$ is a limit ordinal, define $T_{\alpha}:=\lim _{\beta<\alpha} T_{\beta}$. This limit makes sense since at each step described below, either we add some incomplete sub-computation tuples at the end of a computation path that has been introduced at an earlier stage, or turn one incomplete computation tuple in the tree into a complete one.

Thirdly, if $\alpha=\beta+1$, and all computation paths in $T_{\beta}$ are complete, we stop. From now on, assume that the latter is not the case, and also assume that $T_{\beta}$ is well-founded, and thus well-ordered by the Kleene-Brouwer ordering we introduce in the process. Let $\left(t_{0}, \ldots, t_{k}\right)$ be the least element of $T_{\beta}$ in this Kleene-Brouwer ordering consisting of entirely incomplete computation tuples. For our next step, we need to consider the following case distinction.

- If $t_{k}$ is a computation for S1, S2 or S3, i.e. an initial computation. Turn $t_{k}$ into the correct complete version and let $T_{\alpha}$ be the resulting tree. 
- If $t_{k}=\langle e, \vec{a}\rangle$ where $e$ is an index for

$$
\{e\}(F, \mathbf{Y}, \vec{a})=\left\{e_{1}\right\}\left(F, \mathbf{Y},\left\{e_{2}\right\}(F, \mathbf{Y}, \vec{a})\right)
$$

By the choice of $\left(t_{0}, \ldots, t_{k}\right)$ there will be no incomplete extension in the tree $T_{\beta}$. Thus there will be three subcases:

(1) There is no extension of $\left(t_{0}, \ldots, t_{k}\right)$ in $T_{\beta}$ at all. In this case, add $\left(t_{0}, \ldots, t_{k+1}\right)$ to $T_{\beta}$, where $t_{k+1}=\left\langle e_{2}, \vec{a}\right\rangle$.

(2) There is an extension $\left(t_{0}, \ldots, t_{k+1}\right)$ in $T_{\beta}$, where $t_{k+1}=\left\langle e_{2}, \vec{a} ; b\right\rangle$, but no extension of the form $\left(t_{0}, \ldots, t_{k}, t_{k+1}^{\prime}\right)$ where $t_{k+1}^{\prime}=\left\langle e_{1}, b, \vec{a} ; c\right\rangle$. Then add $\left(t_{0}, \ldots, t_{k}, t_{k+1}^{\prime \prime}\right)$ to $T_{\beta}$, where $t_{k+1}^{\prime \prime}=\left\langle e_{1}, b, \vec{a}\right\rangle$. In forming the ordering of $T_{\alpha}$, we let this sub-computation be above the first one in our ordering of sub-computations.

(3) There is an extension $\left(t_{0}, \ldots, t_{k}, t_{k+1}\right)$ of $\left(t_{0}, \ldots, t_{k}\right)$ in $T_{\beta}$ where $t_{k+1}=$ $\left\langle e_{2}, \vec{a} ; b\right\rangle$, and an extension $\left(t_{0}, \ldots, t_{k}, t_{k+1}^{\prime}\right)$ in $T_{\beta}$ where $t_{k+1}^{\prime}=\left\langle e_{1}, b, \vec{a} ; c\right\rangle$. Then obtain $T_{\alpha}$ by replacing $t_{k}$ with $\langle e, \vec{a} ; c\rangle$ in the computation path at hand.

- The cases where $t_{k}$ is a computation for one of the schemes S5 (primitive recursion), S6 (permutation) or S9 (enumeration) are left for the reader as they just will be similar to, or simpler than, the case for S4. Note that S6 does not play a real role, but it gives rise to an initial computation if we allow for function arguments.

- If $t_{k}$ is an S8-computation, given as $t_{k}=\langle e, \vec{a}\rangle$ where

$$
\{e\}(F, \mathbf{Y}, \vec{a})=H(\lambda a .\{d\}(F, \mathbf{Y}, a, \vec{a})),
$$

where either $H$ equals $F$ or $Y_{n}$ for some $n$. There will be two sub-cases:

(1) If $\left(t_{0}, \ldots, t_{k}\right)$ has no extensions in $T_{\beta}$, we extend $T_{\beta}$ to $T_{\alpha}$ by adding all computation paths $\left(t_{0}, \ldots, t_{k}, t_{k+1, a}\right)$ for each $a \in \mathbb{N}$, where $t_{k+1, a}=$ $\langle d, a, \vec{a}\rangle$. We well-order these extensions by the value of $a$.

(2) If $\left(t_{0}, \ldots, t_{k}\right)$ has extensions in $T_{\beta}$, the added computation tuple in all such extensions must be complete, by choice of $\left(t_{0}, \ldots, t_{k}\right)$. Moreover, since item (1) is the only way we add extensions to an S8-computation, there is a function $f$ such that we have an extension with $t_{k+1, a}=$ $\langle d, a, \vec{a} ; f(a)\rangle$ for each $a \in \mathbb{N}$. Now, by choice of $\left(t_{0}, \ldots, t_{k}\right)$ again, if there is any computation path $\left(s_{0}, \ldots, s_{j}\right)$ in $T_{\beta}$ below $\left(t_{0}, \ldots, t_{k}\right)$ in the Kleene-Brouwer ordering, where $s_{j}$ is an S8-computation, $s_{j}$ has to be complete, and some function $g$ has been introduced. If $f$ has already been introduced as some $g$ this way, we know the value of $H(f)$ from before, and use this to make $t_{k}$ complete. If $f$ is introduced for the first time while we replace $T_{\beta}$ with $T_{\alpha}$, we let $n=n(\langle d, \vec{a}\rangle)$ as defined above, we let $f \notin Y_{n}$, and $f \in Y_{m}$ for $m \neq n$, and use this to turn $t_{k}$ into a complete computation tuple for all cases of $H$.

This ends the construction and Definition 6.9 .

We have not said what to do in the case when $T_{\alpha}$ is not well-founded, in which case we cannot identify the least $\left(t_{0}, \ldots, t_{k}\right)$ where all $t_{i}$ are incomplete. Lemma 6.11 shows that this is never the case. The argument is based on Lemma 6.10, which has an easy proof, also under the assumption that the recursion stops when the tree $T_{\alpha}$ is not well-founded. 
Lemma 6.10. For each ordinal $\alpha$ and $\left(t_{0}, \ldots, t_{k}\right) \in T_{\alpha}$, if $t_{k}$ is complete, then $t_{k}$ is the computation tuple of a terminating computation, where $\mathbf{Y}$ is interpreted as the sequence of partial sets defined at stage $\alpha$.

Proof. Trivial, by induction on $\alpha$.

Lemma 6.11. For each ordinal $\alpha, T_{\alpha}$ is a well-founded tree.

Proof. We obviously need a limit ordinal $\alpha$ to introduce an infinite descending sequence. Assume that there is one, and let $\left(t_{0}, t_{1}, \cdots\right)$ be the leftmost one. Let $\mathbf{Y}$ be a total extension of the sequence of partial sets $\lambda n . Y_{n}$ constructed at level $\alpha$. By Lemma 6.10, the sequence will consist only of incomplete computation tuples, where each extension represents a sub-computation. This will be a so-called Moschovakis witness, witnessing that $\left\{e_{0}\right\}(F, \mathbf{Y}, \varepsilon) \uparrow$, which again contradicts the assumption.

We need Lemma6.10 in order to verify that the sequence is indeed a Moschovakis witness when passing an instance of composition (or primitive recursion). Note that in the presence of S9, we do not need the scheme S5, primitive recursion, so for the understanding, one may ignore this case.

We can now complete the proof of Theorem 6.6

Proof. Assume that the theorem is false. Then there is an $F$ and an index $d$ such that for all $\mathbf{Y}$ we have $\xi(\mathbf{Y})=\lambda a .\{d\}(F, \mathbf{Y}, a)$. Let $e_{0}$ be an index such that

$$
\left\{e_{0}\right\}(F, \mathbf{Y}, \varepsilon)=F(\lambda a \cdot\{d\}(F, \mathbf{Y}, a)) .
$$

When we apply our construction above to this $e_{0}$, we construct a $\mathbf{Y}$ where each $Y_{n}$ is open and dense, but where every function $f$ appearing in the computation tree of $\left\{e_{0}\right\}(F, \mathbf{Y}, \varepsilon)$ is left out of exactly one $Y_{n}$. This will in particular be the case for $\lambda a .\{d\}(F, \mathbf{Y}, a)$, so this is not an acceptable value for $\xi(\mathbf{Y})$ after all.

We finish this section with a remark on future research.

Remark 6.12. First of all, the proof of Theorem 6.6 is very different from known proofs of theorems expressing that certain type 3 functionals are not computable in any functional of type 2 ; we refer to [33 35, 37, for the latter kind of proofs.

Secondly, Baire realisers are not unique, but as shown in Theorem 6.5, there is a specimen computable in IND. This begs the question of the necessary complexity of Baire realisers, and how they compare to realisers for HBU and Pincherle's Theorem, as in 33 35, 37. We have no answer to this, and offer it as a research problem.

6.2.2. Reverse Mathematics. We prove the results sketched in items (c) and (d) in Section 6.1. We first introduce our version of the Baire category theorem, called BCT hereafter, based on Definition 1.2 .

Theorem 6.13 (BCT). If $\left(Y_{n}\right)_{n \in \mathbb{N}}$ is a sequence of dense open sets of reals, then $\emptyset \neq \bigcap_{n \in \mathbb{N}} Y_{n}$.

We have the following theorem, establishing that BCT does not exceed $\mathrm{ACA}_{0}$ in terms of first-order strength.

Theorem 6.14. The system $\mathrm{RCA} A_{0}^{\omega}+\mathrm{BOOT}$ proves $\mathrm{BCT}$. 
Proof. In case $\neg\left(\exists^{2}\right)$, all functions on $\mathbb{R}$ are continuous by [27, §3]. Such functions have RM-codes by [26, §4], as $\mathrm{ACA}_{0}$ follows from BOOT. In turn, all open sets now also have RM-codes by [50, II.7.1] and BCT is therefore immediate from the second-order proof of the Baire category theorem (see [50, II.4.10]).

In case $\left(\exists^{2}\right)$, let $Y_{n}$ be as in the theorem and consider the following formula

$$
(\forall y \in \mathbb{R})\left(y \in B\left(q_{m}, r_{m}\right) \rightarrow y \in Y_{n}\right),
$$

which expresses $B\left(q_{m}, r_{m}\right) \subseteq Y_{n}$. Applying BOOT to (6.2) (using $\exists^{2}$ to remove numerical quantifiers), there is $Z \subseteq \mathbb{N} \times \mathbb{N}$ such that $(m, n) \in Z$ if and only if (6.2) holds. Define the sequence $\left(X_{n}\right)_{n \in \mathbb{N}}$ as $\lambda n . Z$ and note that $x \in Y_{n} \leftrightarrow(\exists m \in$ $\mathbb{N})\left(m \in X_{n} \wedge x \in B\left(q_{m}, r_{m}\right)\right)$. Hence, $Y_{n}$ also has an RM-code in this case and BCT is therefore immediate from the aforementioned second-order proof of the Baire category theorem (see [50, II.4.10]).

Let $\mathrm{BOOT}_{\mathrm{w}}$ be BOOT with the (innermost) quantifier over Baire space restricted to $2^{\mathbb{N}}$. As shown in [46, §5], BOOT $w$ has first-order strength at most $\mathrm{WKL}_{0}$, as does the base theory in the following corollary; the proof of the latter is immediate.

Corollary 6.15. The system $\mathrm{RCA}_{0}^{\omega}+\mathrm{BOOT}_{\mathrm{w}}+$ coco proves $\mathrm{BCT}$.

Another corollary of Theorem 6.14 is that $\left(\exists^{3}\right)$ implies BCT, while $\exists^{3}$ computes the real claimed to exist by BCT from the other data. The proof of Theorem 6.14 however also gives rise to the following classical result, where ' $\leq_{T}$ ' means Turing computability. Let $J(Y)=\left\{n \in \mathbb{N}:\left(\exists f \in \mathbb{N}^{\mathbb{N}}\right)(Y(f, n)=0)\right\}$ be the set $X$ as provided by BOOT; there is a term $t$ of Gödel's $T$ such that we have:

for dense open sets $\left(Y_{n}\right)_{n \in \mathbb{N}}$ in $\mathbb{R}$, there is $x \in \cap_{n} Y_{n}$ with $x \leq_{T} J\left(t\left(Y_{n}, \exists^{2}\right)\right)$.

The previous statement can be viewed as the 'second-order' version of the fact that $\exists^{3}$ computes a Baire realiser, in the sense of Kleene S1-S9. We also have the following negative result involving an interesting corollary.

Theorem 6.16. The system $\mathrm{Z}_{2}^{\omega}+\mathrm{QF}-\mathrm{AC}^{0,1}$ cannot prove $\mathrm{BCT}$.

Proof. We shall define a model $\mathcal{M}$ of $Z_{2}^{\omega}+\mathrm{QF}_{-} \mathrm{AC}{ }^{0,1}+\neg \mathrm{BCT}$. Incidentally, $\mathcal{M}$ has been used to establish [37, Theorem 4.3], as well as [38, Theorem 2.2]. The model $\mathcal{M}$ is an element of Gödel's universe $L$ of constructible sets, and the properties will be verified under the assumption that $\mathrm{V}=\mathrm{L}$. Since being a model for the theory in question is absolute, the proof of existence can be formalised in ZF.

Thus, assume $\mathrm{V}=\mathrm{L}$, let $\mathrm{S}_{\omega}^{2}$ be the join of all the functionals $\mathrm{S}_{k}^{2}$, and let $\mathcal{M}$ be the least type-structure closed under computability relative to $S_{\omega}^{2}$. Let $\mathcal{M}_{n}$ be the set of elements in $\mathcal{M}$ of type $n$. We assume $\mathrm{V}=\mathrm{L}$ because then there is a $\Delta_{2}^{1}$-well-ordering of the continuum; this can be used to show that all $\Pi_{k}^{1}$-formulas are absolute for $A=\mathcal{M}_{1}$. Hence, $\mathcal{M}$ is a model for $Z_{2}^{\omega}$ and also satisfies QF-AC ${ }^{0,1}$ as a consequence of Gandy selection for $\mathrm{S}_{\omega}^{2}$.

We now show that $\mathcal{M}_{2}$ contains a functional $F: A \rightarrow \mathbb{N}$ that is injective. This construct is obtained by Gandy selection as follows: for each $f \in A$ we let $F(f)$ be an index for computing $f$ from $S_{\omega}^{2}$. We will use this to show that BCT fails for $\mathbb{N}^{\mathbb{N}}$ in $\mathcal{M}$, but we can use the same idea for Cantor-space or for $\mathbb{R}$ (in $\mathcal{M}$ ).

We now work inside $\mathcal{M}$. Define $O_{n}=\{f: F(f)>n\}$ and note that since the complement of each $O_{n}$ is finite, each $O_{n}$ is open and dense. Moreover, $\{(n, f)$ : 
$\left.f \in O_{n}\right\}$ is definable from $F$ by a term in Gödel's $T$, so this countable sequence of dense, open sets will be in $\mathcal{M}$. The intersection is empty, so BCT fails in $\mathcal{M}$.

The model $\mathcal{M}$ has been used in [38, Theorem 2.2] to show that the statement

$$
(\forall Y:[0,1] \rightarrow \mathbb{N})(\exists x, y \in[0,1])\left(Y(x)={ }_{0} Y(y) \wedge x \neq \mathbb{R} y\right)
$$

cannot be proved in $Z_{2}^{\omega}+$ QF-AC ${ }^{0,1}$. Note that NIN expresses that there is no injection from $[0,1]$ to $\mathbb{N}$. By contrast WHBU $\rightarrow$ NIN and BCT $\rightarrow$ NIN over RCA $_{0}^{\omega}$, as also proved in 38 .

\section{A Finer STUdy OF REPRESENTATIONS OF OPEN SETS}

We establish the results sketched in Section 1.2 regarding the $\Delta$-functional; the latter connects two natural representations of open sets and is introduced in Section 7.1. The main computational properties of the $\Delta$-functional are established in Sections 7.2 and 7.3, while some related RM results are sketched. In a nutshell, these results show that the representation (‥2) of open sets does not have much of an influence on the logical and computational properties of theorems pertaining to open sets, at least in contrast to Definition 1.2

7.1. Introduction. In the previous, we have considered two different representations of open sets, namely the standard (RM) one as in (1.1) and the approach via characteristic functions as in Definition 1.2. There are of course other possible representations, namely as part of (R.1)-(R.4) below; in this section, we show that (R.3) and (‥4) are computationally equivalent, and study the computational properties of the 'conversion' functional $\Delta$ that converts a representation as in (R.2) to a representation as in (R.3). The $\Delta$-functional has interesting properties, as follows.

(P1) $\Delta$ is not computable in any type 2 functional, but computable in any Pincherle realiser, a class weaker than $\Theta$-functionals (Theorem 7.5).

(P2) $\Delta$ is unique, genuinely type 3 , and adds no computational strength to $\exists^{2}$ in terms of computing functions from functions (Corollary 7.6).

Prior to the study of $(\underline{\mathrm{R} .2})$ and $(\underline{\mathrm{R} .3})$, we believed that the only way to find a functional with properties (P1) and (P2) would be through some ad hoc construction and that there would be no natural examples. Regarding RM, we also show that HBU suffices to show that open sets as in (R.2) have a representation as in (R.4).

For the sake of simplicity, we restrict the attention to $[0,1]$, though the $\sigma$ compactness of $\mathbb{R}$ makes it easy to extend all results to $\mathbb{R}$. Thus, we consider the following four ways of representing an open set $O$ in $[0,1]$. For the sake of notational simplicity, we let $(a, b)$ denote $(a, b) \cap[0,1]$. Finally, we could obtain the same results for $2^{\mathbb{N}}$ instead of $[0,1]$, i.e. our results do not really depend on the coding of the real numbers.

(R.1) The set $O$ is $\left\{x \in[0,1] \mid Y(x)>_{\mathbb{R}} 0\right\}$ for some $Y:[0,1] \rightarrow[0,1]$; we just have the extra information that $O$ is open, i.e. as in Definition 1.2 .

(R.2) The set $O$ is represented by a function $Y:[0,1] \rightarrow[0,1]$ such that

(i) we have $O=\left\{x \in[0,1] \mid Y(x)>_{\mathbb{R}} 0\right\}$,

(ii) if $Y(x)>0$, then $(x-Y(x), x+Y(x)) \cap[0,1] \subseteq O$.

(R.3) The set $O$ is represented by the continuous function $Y$ where

(i) $Y(x)$ is the distance from $x$ to $[0,1] \backslash O$ if the latter is nonempty,

(ii) $Y$ is constant 1 if $O=[0,1]$.

(R.4) The set $O$ is given as $\cup_{n \in \mathbb{N}}\left(a_{n}, b_{n}\right)$ for $\left(a_{m}\right)_{m \in \mathbb{N}},\left(b_{m}\right)_{m \in \mathbb{N}}$ sequences in $\mathbb{Q}$. 
Assuming $\exists^{2}$, it is clear that the information given by a representation increases when going down the list. For completeness, we prove that $(\overline{\mathrm{R} .3})$ and $(\overline{\mathrm{R} .4})$ are the same from the computational point of view.

Theorem 7.1. Items ( $(\mathrm{R} .3)$ and ( $\mathrm{R} .4$ ) are computationally equivalent modulo $\exists^{2}$.

Proof. Let $Y$ be continuous as in $(\underline{\mathrm{R} .3})$. Then $Y$ has an RM-code computable in $\exists^{2}$ by [26, §4]. From this representation we can decide if $Y$ is constant 1 or if $Y(x)=0$ for at least one $x$. Let $\alpha$ be this representation for $Y$. Then $x \in O$ if and only if there is some $((a, b),(c, d)) \in \alpha$ such that $c>0$ and $x \in(a, b)$, and the set of intervals $(a, b)$ where $((a, b),(c, d)) \in \alpha$ for some $(c, d)$ with $c>0$ will be a representation of $O$ in the sense of (R.4).

Now assume that $A$ is a set of open rational intervals defining $O$ as in (R.4). Then $O=[0,1]$ if and only if $A$ contains a finite sub-covering of $[0,1]$. If it does, we let $Y$ be the constant 1. If not, let $x$ be given. If $x \notin(a, b)$ for all $(a, b) \in A$, we let $Y(x)=0$. If $x \in(a, b)$ for some $(a, b) \in A$, we let $Y(x)$ be the supremum of the set of rationals $r$ such that $A$ contains a finite sub-covering of $[x-r, x+r]$.

In second-order RM, $\mathrm{ACA}_{0}$ is equivalent to the fact that closed sets are located ([19, Theorem 1.2]). The previous theorem similarly expresses that a set is open if and only if the complement is located. Next, we study the computational relation between the representations defined by $(\underline{\mathrm{R} .2})$ and $(\underline{\mathrm{R} .3})$.

7.2. Converting between representations. In this section, we study the complexity of operators that produce a representation as in (R.3), or equivalently by Theorem 7.1] as in (R.4), from a representation as in (․․2. We (mostly) choose to study (R.3) as this representation is unique for each open set, resulting in a unique functional, i.e. the output of $\Delta$ is unique for equal inputs satisfying (R.3).

Definition 7.2. Let $\Delta^{3}$ be the functional such that $\Delta(Y)$ represents an open set $O$ as in (R.3) whenever $Y$ represents $O$ as in (R.2).

The following proof is straightforward in light of similar proofs in [33 35, 37, and we therefore only provide a sketch.

Lemma 7.3. The functional $\Delta$ is not computable in any type 2 functional.

Proof. Given $F^{2}$, we construct $Y^{2}$ with the following properties.

- The value $Y(f)$ is defined if $f$ represents a fast-converging sequence of rational numbers in $[0,1]$,

- If the sequence represented by $f$ is equivalent to a sequence represented by some $g$ computable in $F$, we use Gandy selection for $F$ to find an index $e$ for one such $g$ as computable in $F$. Note that the Gandy-search is such that the resulting $g$, and index for it, respects equivalence between representations of reals. We then define $Y(f)=2^{-(e+2)}$ for the aforementioned index $e$.

The crux of the previous construction is as follows: the functional $Y$ represents $O=$ $[0,1]$ but no Kleene-algorithm relative to $F$ and $Y$ is able to recognise this. Indeed, since $Y$ is partially computable in $F$ when restricted to functions computable in $F$, it only covers a subset of measure below $\frac{1}{2}$ in this situation.

Next, we show that $\Delta$ is computable from $\exists^{2}$ and a Pincherle realiser, i.e. a realiser for Pincherle's theorem, a concept first introduced in [37. The latter theorem expresses that a locally bounded functional on $C$ is also bounded (see [40, p. 67]); 
a Pincherle realiser (PR for short) computes this upper bound in terms of some of the other data. We consider the following equivalent form of Pincherle realisers, going back to an equivalent formulation by Pincherle himself (see [37, 40]). Note that we assume that $Y$ is extensional on the reals.

Definition 7.4. A $P R$ is any functional $M_{u}^{3}$ such that for any $Y:[0,1] \rightarrow \mathbb{R}^{+}$, the number $M_{u}(Y)>_{\mathbb{R}} 0$ is a lower bound for all $Z:[0,1] \rightarrow \mathbb{R}$ locally bounded away from zero by $Y$, i.e. satisfying $(\forall x, y \in[0,1])(|x-y|<Y(x) \rightarrow Z(y)>Y(x))$.

Note that the functional $Y$ is a realiser for ' $Z$ is locally bounded from zero'. As discussed in [37, Pincherle assumes the existence of such realisers (for local boundedness) in [40, p. 66]. The following theorem is interesting, as PRs are weaker than $\Theta$-functionals (realisers for $\mathrm{HBU}$ ), as shown in [37.

Theorem 7.5. The functional $\Delta$ is computable in any $P R$ and $\exists^{2}$.

Proof. Let $Y$ represent the open set $O$ as in (‥2. $)$. We first show that any PR $M_{u}$ and $\exists^{2}$ allows us to decide if $O=[0,1]$ or not.

Define $Y_{n}(x)$ as $Y(x)$ if $Y(x)_{\mathbb{R}}>0$ and $2^{-n}$ otherwise. If $O=[0,1]$ then $Y_{n}=Y$ for all $n$, and $M_{u}\left(Y_{n}\right)$ is positive and independent of $n$. If $x_{0} \notin O$, there is no $x$ such that $Y(x)>0$ and $x_{0}$ is in the neighbourhood around $x$ defined by $Y(x)$. Hence, $2^{-n}$ will be the lower bound on $Z\left(x_{0}\right)$ induced by $Y_{n}$, yielding $0<M_{u}\left(Y_{n}\right) \leq 2^{-n}$. Hence, we can decide if $O=[0,1]$ or not using the sequence $\lambda n . M_{u}\left(Y_{n}\right)$.

Next consider $x \in[0,1]$ and assume that there is some (unknown) $z \notin O$. For each rational $r>0$, we define the following set:

$$
\left.O_{x, r}=O \cup\{y \in[0,1]: \mid x-y]>r\right\},
$$

and we let $Y_{x, r}$ be the representation of $O_{x, r}$ provided by (․2). Now let $Z(x)$ be the distance from $x$ to the complement of $O$. Then $Z(x) \leq r$ if and only if $O_{x, r} \neq[0,1]$, and we can use $M_{u}$ as above deciding this for each $x \in[0,1]$ and $r$. Since we can decide if $Z(x) \leq r$ uniformly in $r$, we can use $\exists^{2}$ to compute $Z(x)$.

We have the following corollary, establishing the above claims concerning the $\Delta$-functional introduced in the previous section.

Corollary 7.6. The functional $\Delta$ is computationally 'weak' as follows.

(a) For each $f \in \mathbb{N}^{\mathbb{N}}$, all functions computable in $\Delta, \exists^{2}, f$ are also hyperarithmetical in $f$ alone.

(b) There is no PR $M_{u}$ computable in $\Delta$ and $\exists^{2}$.

Proof. Since any $\Theta$-functional computes some PR, $\Delta$ is uniformly computable in any $\Theta$-functional, and the only functions that are uniformly computable in any $\Theta$ and $\exists^{2}$ are the hyperarithmetical ones (see [35,37]). This readily relativises to any function $f$, i.e. the first item follows.

Each PR $M_{u}$ computes (non-uniformly) a non-hyperarithmetical function, while $\Delta$ only computes hyperarithmetical ones, i.e. no $M_{u}$ can be computable in $\Delta$.

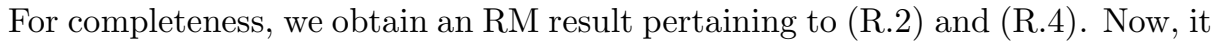
is shown in 37] that a uniform version of Pincherle's theorem (which forms the basis for PRs) and HBU are equivalent over a weak base theory. In light of Theorem 7.5 . one therefore expects HBU to suffice to prove the following coding principle, as shown in Theorem 7.8. Corollary 7.6 suggests no reversal can be obtained. 
Definition 7.7. [open ${ }^{-}$] An open set as in (R.2) has a representation as in (R.4).

The following theorem should be contrasted with [46, Theorem 4.4] where it is shown that a generalisation of open and open ${ }^{-}$implies BOOT (which implies HBU). Note that the following proof makes explicit use of the extra information that R.2 includes over (R.1), i.e. the latter cannot be treated in the same way.

Theorem 7.8. The system $\mathrm{RCA}_{0}^{\omega}+\mathrm{HBU}+\mathrm{QF}-\mathrm{AC}^{0,1}$ proves open ${ }^{-}$.

Proof. We may assume $\left(\exists^{2}\right)$ as the theorem is trivial in case $\neg\left(\exists^{2}\right)$. Indeed, all functions on $\mathbb{R}$ are continuous in this case by [27, §3], while WKL suffices to provide RM-codes on $[0,1]$ by [26, $\$ 4]$. Then [50, II.7.1] yields the required RM-open set.

Let $O$ and $Y$ be as in (R.2) and consider the following for any $n \in \mathbb{N}, q \in \mathbb{Q} \cap[0,1]$ :

$$
\bar{B}\left(q, \frac{1}{2^{n}}\right) \subset O \leftrightarrow\left(\exists y_{0}, \ldots, y_{k} \in O\right)\left[\bar{B}\left(q, \frac{1}{2^{n}}\right) \subset \cup_{i \leq k} B\left(y_{i}, Y\left(y_{i}\right)\right)\right],
$$

where $\bar{B}(x, r)$ is the closed ball with center $x$ and radius $r>_{\mathbb{R}} 0$. The reverse implication in (7.1) follows by definition. The forward implication in (7.1) follows from applying $\mathrm{HBU}$ to the uncountable covering $\cup_{x \in \bar{B}\left(q, \frac{1}{2^{n}}\right)} B(x, Y(x))$ of $\bar{B}\left(q, \frac{1}{2^{n}}\right)$.

Now note that the formula in big square brackets in (7.1) is decidable thanks to $\exists^{2}$, while $\bar{B}\left(q, \frac{1}{2^{n}}\right) \subset O$ ' has the form $(\forall y \in \mathbb{R}) B(y, q, n)$ where $B$ is also decidable modulo $\exists^{2}$. In other words, (7.1) is equivalent to a formula of the form $\left(\forall m^{0}\right)((\forall y \in \mathbb{R}) B(y, m) \leftrightarrow(\exists x \in \mathbb{R}) A(x, m))$. Clearly, QF-AC ${ }^{0,1}$ applies to $\left(\forall m^{0}\right)((\forall y \in \mathbb{R}) B(y, m) \rightarrow(\exists x \in \mathbb{R}) A(x, m))$, yielding $\Phi^{0 \rightarrow 1}$ such that $\left(\forall m^{0}\right)((\forall y \in$ $\mathbb{R}) B(y, m) \leftrightarrow(\exists x \in \mathbb{R}) A(x, m)) \leftrightarrow A(\Phi(m), m)$. In this way, there is a set $X \subset \mathbb{Q} \times \mathbb{N}$ such that for any $q \in \mathbb{Q} \cap[0,1], n \in \mathbb{N}$, we have

$$
(q, n) \in X \leftrightarrow\left(\exists y_{0}, \ldots, y_{k} \in O\right)\left[\bar{B}\left(q, \frac{1}{2^{n}}\right) \subset \cup_{i \leq k} B\left(y_{i}, Y\left(y_{i}\right)\right)\right] .
$$

Again modulo $\exists^{2}$, apply QF-AC $C^{0,1}$ to the forward implication in (7.1) to find $\Psi^{0 \rightarrow 1^{*}}$ such that if $(q, n) \in X, \Psi(q, n)=\left\langle y_{0}, \ldots, y_{k}\right\rangle$ is as in the right-hand side of (7.2). Now consider the following formula based on the previous:

$$
x \in O \leftrightarrow(\exists n \in \mathbb{N})(\exists q \in \mathbb{Q} \cap[0,1])\left((q, n) \in X \wedge x \in O_{q, n}\right),
$$

where $O_{q, n}$ is $\cup_{i<|\Psi(q, n)|} B(\Psi(q, n)(i), Y(\Psi(q, n)(i)))$. The reverse implication in (17.3) follows by (7.1) and (7.2). For the forward implication in (7.3), fix $x_{0} \in O$ and let $n_{0}$ be large enough to guarantee $\bar{B}\left(q_{0}, \frac{1}{2^{n_{0}}}\right) \subset O$ for $q_{0}:=\left[x_{0}\right]\left(n_{0}\right)$. By (17.1) and (17.2), we have $\left(q_{0}, n_{0}\right) \in X$ and thus $\bar{B}\left(q_{0}, \frac{1}{2^{n} 0}\right) \subset O_{q_{0}, n_{0}}$. By assumption, $x_{0}$ is in $\bar{B}\left(q_{0}, \frac{1}{2^{n_{0}}}\right)$ and (7.3) thus provides the countable union required by (R.4).

We note that (7.2) is a special case of a new comprehension axiom called ' $\Delta$ comprehension' or ' $\triangle$-CA' in [46 48; this axiom yields the well-known recursive comprehension axiom from $\mathrm{RCA}_{0}$ under ECF. There is no connection to the $\Delta$ functional beyond a shared symbol. We also note that HBU can be replaced by the Lindelöf lemma for $\mathbb{R}$, as studied in [37].

As a conceptual corollary, the previous theorem shows that the RM of HBU generalises to theorems pertaining to open and closed sets as in (R.2).

7.3. Representations, Heine-Borel, and Baire. We establish two theorems on the computational complexity of the countable Heine-Borel theorem and the Baire category theorem when formulated using the representation ( R.2).

First of all, $\Delta+\exists^{2}$ suffices to compute finite sub-coverings for coverings formulated using $(\underline{\mathrm{R} .2})$; no type two functional can replace $\Delta$ here. 
Theorem 7.9. Let $Y_{n}$ be a representation of the open set $O_{n} \subseteq[0,1]$ as in $(\mathbb{R} .2)$ and assume $[0,1] \subseteq \cup_{n \in \mathbb{N}} O_{n}$. Then $\Delta+\exists^{2}$ computes $k \in \mathbb{N}$ such that $[0,1] \subseteq \cup_{i \leq k} O_{i}$. The functional $\Delta$ cannot be replaced by any functional of type 2 here.

Proof. Using $\Delta$ on each $Y_{n}$, and the equivalence between ( $\left.\mathrm{R} .3\right)$ and (R.4 $)$, we obtain a standard RM-covering of the unit interval, and then we only need a realiser for WKL, computable in $\exists^{2}$, to prove the first claim.

For the second claim, we use one of our standard techniques: given $F^{2}$, assume that the realiser $\beta$ for HBC (formulated with $(\overline{\mathrm{R} .2})$ ) is computable in $F+\exists^{2}$. We let each $Y_{n}$ be the same: it represent $[0,1]$ as an open set but in such a way that $Y_{n}$ restricted to the reals computable in $F+\exists^{2}$ is partially computable in $F+\exists^{2}$ and only defines an open set of measure $\leq \frac{1}{2}$. Let $k=\beta\left(\lambda n . Y_{n}\right)$, and let $Y_{i}^{\prime}$ be derived from $Y_{i}$ for $i \leq k+1$ by removing one, and the same, point that is outside this set. Let $Y_{i}^{\prime}=Y_{i}$ for $i>k+1$. Then $\beta\left(\lambda n . Y_{n}\right)=\beta\left(\lambda n . Y_{n}^{\prime}\right)$, contradicting what $\beta$ is supposed to achieve.

The first part of this proof also implies that the optimal realiser for HBC (formulated using $(\underline{\mathbb{R} .2})$ ), the one selecting the least $k$ as in Theorem 7.9 , is equivalent to $\Delta$, given $\exists^{2}$. We leave the proof of this to the reader.

Secondly, the Baire category theorem becomes a lot 'tamer' from the computational point of view upon the introduction of (․2.2).

Theorem 7.10. Assuming a representation as in (R.2) is given for all the $Y_{n}$, we can compute a Baire realiser relative to $\exists^{2}$. If we know that each $Y_{n}$ represents a dense open set, we can even avoid $\exists^{2}$.

Proof. Let $Y_{n}$ be given for each $n$ and first assume that each $Y_{n}$ is an (‥2) representation of the open, dense set $O_{n}$. Then the text-book proof of the Baire category theorem can be transformed to an algorithm by using $Y_{n}$ restricted to the rational numbers to find the shrinking sequence of open-and then-closed intervals, and iterated search over $\mathbb{Q}$ for the sequence that converges to a point in the intersection.

We need to search for $q \in \mathbb{Q}$ within an open interval such that $Y_{n}(q)>0$, but since the latter relation is $\Sigma_{1}^{0}$, this is effective. If one $Y_{n}$ does not represent an open, dense set, the only thing that might prevent this algorithm from terminating is that the search goes on for ever, and whether this will be the case can be decided using $\exists^{2}$, so we can output a value even then. In this case, it does not matter what the value is, we are only required to have one.

Acknowledgement 7.11. Our research was supported by the John Templeton Foundation via the grant a new dawn of intuitionism with ID 60842 and by the Deutsche Forschung Gemeinschaft (DFG) via grant SA3418/1-1. The results in Section 4 were completed during the stimulating BIRS workshop (19w5111) on Reverse Mathematics at CMO, Oaxaca, Mexico in Sept. 2019. We express our gratitude towards the aforementioned institutions. We thank Anil Nerode and the referees for their helpful suggestions. Opinions expressed in this paper do not necessarily reflect those of the John Templeton Foundation.

\section{REFERENCES}

[1] Peter Aczel, A constructive version of the Lusin separation theorem, Logicism, intuitionism, and formalism, Synth. Libr., vol. 341, Springer, Dordrecht, 2009, pp. 129-151. 
[2] Jeremy Avigad and Solomon Feferman, Gödel's functional ("Dialectica") interpretation, Handbook of proof theory, Stud. Logic Found. Math., vol. 137, 1998, pp. 337-405.

[3] Vasco Brattka, Guido Gherardi, and Rupert Hölzl, Probabilistic computability and choice, Inform. and Comput. 242 (2015), 249-286.

[4] Vasco Brattka, Matthew Hendtlass, and Alexander P. Kreuzer, On the uniform computational content of the Baire category theorem, Notre Dame J. Form. Log. 59 (2018), no. 4, 605-636.

[5] Errett Bishop, Foundations of constructive analysis, McGraw-Hill, 1967.

[6] E. Borel, Sur la classification des ensembles de mesure nulle, Bull. Soc. Math. France 47 (1919), 97-125.

[7] Nicolas Bourbaki, Elements of the history of mathematics, Springer, 1994.

[8] Douglas K. Brown, Functional analysis in weak subsystems of second-order arithmetic, $\mathrm{PhD}$ Thesis, The Pennsylvania State University, ProQuest LLC, 1987.

[9] - Notions of closed subsets of a complete separable metric space in weak subsystems of second-order arithmetic, Logic and computation (Pittsburgh, PA, 1987), Contemp. Math., vol. 106, Amer. Math. Soc., Providence, RI, 1990, pp. 39-50.

[10] - Notions of compactness in weak subsystems of second order arithmetic, Reverse mathematics 2001, Lect. Notes Log., vol. 21, Assoc. Symbol. Logic, 2005, pp. 47-66.

[11] Wilfried Buchholz, Solomon Feferman, Wolfram Pohlers, and Wilfried Sieg, Iterated inductive definitions and subsystems of analysis: recent proof-theoretical studies, LNM 897, Springer, 1981.

[12] Constantin Carathéodory, Vorlesungen über reelle Funktionen, Teubner, 1918 (German).

[13] Pierre Cousin, Sur les fonctions de $n$ variables complexes, Acta Math. 19 (1895), 1-61.

[14] Richard Dedekind, Gesammelte mathematische Werke. Bände II, Herausgegeben von Robert Fricke, Emmy Noether und öystein Ore, Chelsea Publishing Co., New York, 1968 (German).

[15] Solomon Feferman, How a Little Bit goes a Long Way: Predicative Foundations of Analysis, 2013. https://math.stanford.edu/ feferman/papers/pfa.pdf

[16] Harvey Friedman, Some systems of second order arithmetic and their use, Proceedings of the International Congress of Mathematicians (Vancouver, B. C., 1974), Vol. 1, 1975, pp. 235-242.

[17] — Systems of second order arithmetic with restricted induction, I \& II (Abstracts), Journal of Symbolic Logic 41 (1976), 557-559.

[18] Robin Gandy, General recursive functionals of finite type and hierarchies of functions, Ann. Fac. Sci. Univ. Clermont-Ferrand No. 35 (1967), 5-24.

[19] Mariagnese Giusto and Stephen G. Simpson, Located sets and reverse mathematics, J. Symbolic Logic 65 (2000), no. 3, 1451-1480.

[20] John P. Hartley, The countably based functionals, J. Symbolic Logic 48 (1983), no. 2, 458-474.

[21] Horst Herrlich, Axiom of choice, Lecture Notes in Mathematics, vol. 1876, Springer, 2006.

[22] David Hilbert and Paul Bernays, Grundlagen der Mathematik. II, Zweite Auflage. Die Grundlehren der mathematischen Wissenschaften, Band 50, Springer, 1970.

[23] James Hunter, Higher-order reverse topology, ProQuest LLC, Ann Arbor, MI, 2008. Thesis (Ph.D.)-The University of Wisconsin - Madison.

[24] Kyriakos Keremedis, Disasters in topology without the axiom of choice, Arch. Math. Logic 40 (2001), no. 8.

[25] Stephen C. Kleene, Recursive functionals and quantifiers of finite types. I, Trans. Amer. Math. Soc. 91 (1959), 1-52.

[26] Ulrich Kohlenbach, Foundational and mathematical uses of higher types, Reflections on the foundations of mathematics, Lect. Notes Log., vol. 15, ASL, 2002, pp. 92-116.

[27] Higher order reverse mathematics, Reverse mathematics 2001, Lect. Notes Log., vol. 21, ASL, 2005, pp. 281-295.

[28] Henri Lebesgue, Sur le probléme de Dirichlet, Rendiconti del Circolo Matematico di Palermo 24 (1907), no. 1, 371-402.

[29] John Longley and Dag Normann, Higher-order Computability, Theory and Applications of Computability, Springer, 2015.

[30] Y. Moschovakis, Hyperanalytic predicates, Trans. Amer. Math. Soc. 129 (1967), 249-282.

[31] Gregory H. Moore, The emergence of open sets, closed sets, and limit points in analysis and topology, Historia Math. 35 (2008), no. 3, 220-241.

[32] P. Muldowney, A general theory of integration in function spaces, including Wiener and Feynman integration, Vol. 153, Longman Scientific \& Technical, Harlow; John Wiley, 1987. 
[33] Dag Normann and Sam Sanders, Nonstandard Analysis, Computability Theory, and their connections, Journal of Symbolic Logic 84 (2019), no. 4, 1422-1465.

[34] - The strength of compactness in Computability Theory and Nonstandard Analysis, Annals of Pure and Applied Logic, Article 102710170 (2019), no. 11.

[35] _ On the mathematical and foundational significance of the uncountable, Journal of Mathematical Logic, https://doi.org/10.1142/S0219061319500016 (2019).

[36] _ Representations in measure theory, Submitted, arXiv: https://arxiv.org/abs/1902.02756 (2019).

[37] - Pincherle's theorem in Reverse Mathematics and computability theory, Annals of Pure and Applied Logic, doi: 10.1016/j.apal.2020.102788 (2020).

[38] - On the uncountability of $\mathbb{R}$, Submitted, arxiv: https://arxiv.org/abs/2007.07560 (2020).

[39] William Fog Osgood, Non-Uniform Convergence and the Integration of Series Term by Term, Amer. J. Math. 19 (1897), no. 2, 155-190.

[40] Salvatore Pincherle, Sopra alcuni sviluppi in serie per funzioni analitiche (1882), Opere Scelte, I, Roma (1954), 64-91.

[41] Nobuyuki Sakamoto and Takeshi Yamazaki, Uniform versions of some axioms of second order arithmetic, MLQ Math. Log. Q. 50 (2004), no. 6, 587-593.

[42] Sam Sanders and Keita Yokoyama, The Dirac delta function in two settings of Reverse Mathematics, Archive for Mathematical Logic 51 (2012), no. 1, 99-121.

[43] Sam Sanders, The Gandy-Hyland functional and a computational aspect of Nonstandard Analysis, Computability 7 (2018), 7-43.

[44] _ Reverse Mathematics of topology: dimension, paracompactness, and splittings, To appear in Notre Dame Journal for Formal Logic, arXiv:/https://arxiv.org/abs/1808.08785 (2019), pp. 21.

[45] - Nets and Reverse Mathematics: a pilot study, Computability, doi:10.3233/COM-190265 (2019), pp. 34 .

[46] Plato and the foundations of mathematics, Submitted, arxiv: https://arxiv.org/abs/1908.05676 (2019), pp. 40.

[47] Lifting countable to uncountable mathematics, To appear in Information and Communication, arxiv: https://arxiv.org/abs/1908.05677 (2019), pp. 21.

[48] _ Lifting recursive counterexamples to higher-order arithmetic, Proceedings of LFCS2020, Lecture Notes in Computer Science, Springer (2020).

[49] Stephen G. Simpson (ed.), Reverse mathematics 2001, Lecture Notes in Logic, vol. 21, ASL, La Jolla, CA, 2005.

[50] - Subsystems of second order arithmetic, 2nd ed., Perspectives in Logic, CUP, 2009.

[51] Richard A. Shore, Reverse mathematics, countable and uncountable, Effective mathematics of the uncountable, Lect. Notes Log., vol. 41, Assoc. Symbol. Logic, La Jolla, CA, 2013, pp. $150-163$.

[52] John Stillwell, Reverse mathematics, proofs from the inside out, Princeton Univ. Press, 2018.

[53] Charles Swartz, Introduction to gauge integrals, World Scientific, 2001.

[54] Terence Tao, Compactness and Compactification (Timothy Gowers, ed.), The Princeton Companion to Mathematics, Princeton University Press, 2008.

[55] Heinrich Tietze, Über Funktionen, die auf einer abgeschlossenen Menge stetig sind, J. Reine Angew. Math. 145 (1915), 9-14.

[56] Anne Sjerp Troelstra, Metamathematical investigation of intuitionistic arithmetic and analysis, Springer Berlin, 1973. Lecture Notes in Mathematics, Vol. 344.

[57] Anne Sjerp Troelstra and Dirk van Dalen, Constructivism in mathematics. Vol. I, Studies in Logic and the Foundations of Mathematics, vol. 121, North-Holland, 1988.

[58] Charles-Jean de La Vallée Poussin, Intégrales de Lebesgue, Guathier-Villars, 1916.

[59] C. T. Chong, Wei Li, Wei Wang, and Yue Yang, On the computability of perfect subsets of sets with positive measure, Proc. Amer. Math. Soc. 147 (2019), no. 9.

[60] Klaus Weihrauch and Christoph Kreitz, Representations of the real numbers and of the open subsets of the set of real numbers, Ann. Pure Appl. Logic 35 (1987), no. 3, 247-260.

[61] Klaus Weihrauch, Computable analysis, Springer-Verlag, Berlin, 2000. An introduction.

[62] Xiaokang Yu, Measure theory in weak subsystems of second-order arithmetic, ProQuest LLC, Ann Arbor, MI, 1987. Thesis (Ph.D.)-The Pennsylvania State University. 